\title{
PRESSURE-SHIFT NUCLEATION: A POTENTIAL TOOL FOR FREEZE CONCENTRATION OF FLUID FOODS
}

\author{
Otero, L.*; Sanz, P., Guignon, B. and Sanz, P. D. \\ Malta Consolider Team. \\ Department of Processes, Institute of Food Science, Technology and Nutrition (ICTAN-CSIC) \\ c/ José Antonio Nováis, 10, 28040 Madrid, Spain
}

\begin{abstract}
Pressure-shift nucleation (PSN) has been evaluated as a potential substitute of the crystallization step at the scraped surface heat exchanger in conventional freeze concentration. To do that, PSN experiments were carried out at different pressure and temperature conditions in orange juices of several concentrations. After crystallization, the final concentration reached and the size and shape of the ice crystals formed were measured. The results obtained showed that the higher the pressure and the lower the temperature employed in the PSN experiments, the higher is the final concentration in the juice and the smaller the ice crystals formed. Four important advantages of pressure-shift nucleation over conventional crystallization were found: temperature in the pressure vessel can be relatively high if pressure is increased enough, the desired concentration can be achieved in the whole sample quasi-instantaneously just after expansion, ice crystals produced are round in shape without pockets and indentations and they are homogeneously distributed throughout the sample.
\end{abstract}

Keywords: high-pressure; freeze concentration; nucleation; supercooling; ice crystals; orange juice

\section{INTRODUCTION}

Concentration of fluid foods is a separation process in which water is removed from the product. It is a common operation in the food industry because it involves a volume reduction in the processed products which allows important savings in transport, storage and packing costs. In addition, it increases the stability and shelf life of food by reducing its water activity (Deshpande, Cheryan, Sathe, Salunkhe, \& Luh, 1984; Ramteke, Singh, Rekha, \& Eipeson, 1993).

Among all the existing methods for food concentration, freeze concentration, also named cryoconcentration, is the most advantageous technique to obtain high quality products

* Corresponding author. Tel.: +34 9154456 07; fax : +34 915493627 .

E-mail address: l.otero@ictan.csic.es (L. Otero). 
without appreciable loss in taste, aroma, color, or nutritive value. In freeze concentration, water is removed from a liquid food by cooling it until ice crystals are formed. These crystals are, then, physically separated to leave behind a more concentrated fluid. Ice is formed as pure water crystals and, therefore, the process provides a high selectivity toward water removal. Since all the process is carried out in an enclosed system and below freezing temperatures, chemical deterioration and microbiological and enzymatic activity in the food are very low and no loss of volatile aroma is produced (Braddock \& Marcy, 1985; Deshpande et al., 1984). Nowadays, these are very important advantages when consumer preferences point toward high quality products that, while having longer shelf-life than the fresh ones, have similar sensorial attributes and nutritional value to the original product. However, despite these advantages, freeze concentration is hardly employed in the food industry mainly due to economic aspects of the technology.

Several freeze concentration techniques have been conceived to improve the effectiveness of the concentration process: suspension crystallization, progressive cryoconcentration, eutectic cryoconcentration and partial or complete block cryoconcentration (Aider \& de Halleux, 2009; Deshpande et al., 1984; Hernández, Raventós, Auleda, \& Ibarz, 2010; Rahman, Ahmed, \& Chen, 2006). Nevertheless, in the last five decades, only suspension crystallization has been successfully implemented in the food industry under different solutions: Daubron Process, Gasquet Process, Phillips Process, Struthers FreCon Method, Union Carbide Process or Grenco Process, among others (Deshpande et al., 1984). Grenco Process, also known as traditional Niro Freeze Concentration Technology ${ }^{\circledR}$, is the patented technology more widely employed at the beverage industry, with more than 60 freeze concentration plants throughout the world (PT, 2010). It is based on the separate nucleation and growth concept, that is, the areas for ice crystal production and ice crystal growth must be separated because the optimal operating conditions for these distinct crystallization phenomena are significantly different (Rahman et al., 2006; Van Nistelrooij, 2005). This technology involves a rather complicated processing system composed of a scraped surface heat exchanger (SSHE) for the generation of ice crystals, a re-crystallization vessel for ice crystal growth and a wash column for separation. The product to be concentrated is pumped from a feed tank into the scraped surface heat exchanger where small dendritic ice crystals $(1-10 \mu \mathrm{m})$ are continuously formed on the walls. Mechanical scrapers remove these crystals which are, subsequently, pumped to the re-crystallization tank. Here, they grow, mixed with large crystals, to reach an average particle size of about 300-500 $\mu \mathrm{m}$ (Van Nistelrooij, 2005). Then, the slurry mixture of concentrate and ice crystals is transported to the wash column and the concentrate is subsequently "squeezed" through a filter. Finally, the ice crystals are washed in the column to eliminate the concentrate occluded in the ice cake and the final concentrate is pumped to the storage vessel or to the next stage in the production process.

The scraped surface heat exchanger is the most expensive processing unit in the Niro Freeze Concentration Technology ${ }^{\circledR}$ due to its high capital cost, roughly $30 \%$ of the total investment costs in a freeze concentration plant, and to its relatively high power consumption 
(Habib \& Farid, 2006). In the SSHE, ice nucleation only occurs at the refrigerated walls and the product must be continuously circulating to progressively reach the desired concentration level. To make the process efficient, high flow rates and low residence times must be set at the SSHE and, therefore, low temperatures which allow high thermal gradients and high supercooling must be employed. Operation costs are due not only to the low temperatures needed but also to the expense of electricity required for the continuous rotation of the scraping blades. Moreover, the dendritic ice crystals formed are not appropriate to be efficiently separated at the wash column because they are too small and they have pockets and indentations in which the concentrated solution might be trapped. Therefore, a re-crystallization step is required to make the separation process easier.

Many efforts have been made to overcome all these drawbacks of the scraped surface heat exchanger and improve the crystallization step. Different alternatives, like slurry crystallization (Van Nistelrooij, 2005) or fluidized-bed heat exchangers (Habib \& Farid, 2006) have been proposed to minimize costs. Also, vacuum crystallization (Verschuur, Scholz, Van Nistelrooij, \& Schreurs, 2002) and the use of ultrasound (Botsaris \& Qian, 1999) have been suggested to better control ice nucleation and crystallization, but up to date, none of these alternatives have been commercially implemented.

In this paper, the use of high-pressure as a tool to enhance and control ice nucleation in freeze concentration is presented. In the last decades, high-pressure shift freezing has been proved to be an interesting freezing technology as compared to traditional freezing methods (Cheftel, Lévy, \& Dumay, 2000; LeBail, Chevalier, Mussa, \& Ghoul, 2002; Sanz \& Otero, 2005). Pressure-shift nucleation (PSN) is the key step which could be exploited in freeze concentration. In brief, the sample is cooled to subzero temperatures under pressure to avoid ice nucleation. Once the target temperature is reached, it is subjected to a sudden pressure release. This induces a high and uniform supercooling in the whole product, whichever its size or shape, and a large percentage of water is quasi-instantaneously crystallized throughout it and not only at its surface as occurs in conventional freezing (Cheftel et al., 2000; Otero \& Sanz, 2000, 2006). In this way, nucleation is perfectly controlled because it is only triggered after the pressure release which is a controllable event. Moreover, the amount of ice formed can be adjusted since it mainly depends on the pressure and temperature conditions before expansion (Otero \& Sanz, 2000, 2006). On the other hand, ice crystals formed in high-pressure shift freezing have been described as granular, with no indentations, and dispersed throughout the sample (Kanda, Aoki, \& Kosugi, 1992; Lévy, Dumay, Kolodziejczyk, \& Cheftel, 1999; Martino, Otero, Sanz, \& Zaritzky, 1998). All these reported antecedents indicate that pressureshift nucleation could be employed as a substitute of the crystallization step at the scraped surface heat exchanger in freeze concentration. Nevertheless, no experimental studies have been made, up to date, to assess the viability of this idea.

The aim of this paper is to assess the feasibility of pressure-shift nucleation as a substitute of the crystallization step at the SSHE in conventional freeze concentration. To do that, PSN experiments were carried out at different pressure and temperature conditions in 
orange juice, a liquid food commonly concentrated in the food industry. After crystallization, the final concentration reached and the size and shape of the ice crystals formed were measured. The effect of some process parameters like the initial concentration of the sample or the pressure and temperature conditions employed was also analyzed in depth. From these results, a model to predict the final concentration in the juice was developed and different modes to implement pressure-shift nucleation in a freeze concentration process were proposed. All these data can provide important information to evaluate the feasibility of high-pressure nucleation for cryoconcentration.

\section{MATERIALS AND METHODS}

\subsection{Samples}

Reconstituted orange juice samples of 11.8 (representing fresh single-strength juice), 20, 30 and 40 Brix were prepared from a commercial frozen orange juice concentrate (NUFRI, Lérida, Spain). To obtain samples with different water contents, the commercial concentrate (66.5 ํㅏix) was diluted with distilled water. Brix levels were measured using a digital refractometer (Leica AR200, Leica Microsystems Inc, New York, USA) with automatic temperature compensation.

\subsection{Pressure-shift nucleation experiments}

PSN experiments were performed at four different pressure conditions (200, 350, 500 and $700 \mathrm{MPa})$ and two temperatures $(-5 \stackrel{\circ}{\circ}$ and $-20 \stackrel{\circ}{\circ})$. These pressure and temperature conditions were chosen to cover the minimum and maximum pressure and temperature levels at which the cryoconcentration process could be of interest. Working at pressures lower than $200 \mathrm{MPa}$ or at temperatures higher than $-5{ }^{\circ} \mathrm{C}$ would render negligible amounts of ice while working at pressures higher than $700 \mathrm{MPa}$ or at temperatures lower than $-20{ }^{\circ} \mathrm{C}$ would induce undesired freezing of the sample under pressure. For this reason, to avoid undesired ice formation under pressure, experiments at $-20{ }^{\circ} \mathrm{C}$ were only performed at 200 and $350 \mathrm{MPa}$.

Figure 1 shows the pressure/temperature coordinates from which expansions were made over the phase diagram of pure water (Wagner, Saul, \& Pruss, 1994). Melting curves of orange juice samples (dotted lines in Figure 1) were estimated according to the linear additive model (Guignon et al., 2008) and the equilibrium freezing curve proposed by Chen et al. (1990) for orange juice at atmospheric conditions. Points in Figure 1 are expected to show the effects of pressure and temperature on the final concentration achieved after expansion and on the size and shape of the ice crystals formed. Note that coordinates $350 \mathrm{MPa} /-20 \stackrel{\circ}{\circ}$ and $700 \mathrm{MPa} /-$ $5 \stackrel{\circ}{\circ}$ are below the melting curve of some or all of the juices employed. Previous experiments showed that undesired freezing at both conditions does not occur because nucleation under pressure requires high supercooling to take place (Knorr, Schlüter, \& Heinz, 1998; Otero \& Sanz, 2006). 
Experiments were carried out in a lab-scale high-pressure equipment U111 (Institute of High Pressure Physics, Unipress Equipment Division, Poland) designed to work at pressures up to $700 \mathrm{MPa}$ and temperatures between $-40^{\circ} \mathrm{C}$ and $100^{\circ} \mathrm{C}$. It was composed of a CuBe alloy vessel and an electric power and control unit (X US/2003-Unipress, Poland). Pressure was generated by a high-pressure intensifier driven by a hydraulic power unit $(01 / 5200145$, Rexroth Bosch Group Ltd, Poland). Dimensions of the vessel were internal diameter $30 \mathrm{~mm}$, height 64 $\mathrm{mm}$ and working volume $45 \mathrm{ml}$. Silicon oil M40.165.10 (Peter Huber Kältemaschinenbau GmbH, Offenburg, Germany) was used as compressing fluid. Homogeneous temperature in the inner volume of the vessel and quick temperature equilibration after pressure build-up were achieved by immersing the vessel in a thermostatic tank filled with cold ethanol. Ethanol was continuously circulating between this tank and a thermostatic bath (Haake F3-K, Fisons Instruments, Karlsruhe, Germany) and it was maintained $0.5{ }^{\circ} \mathrm{C}$ under the target temperature. This small difference between the ethanol temperature and the target temperature results in long cooling times under pressure, but it is necessary to have a homogeneous temperature throughout the sample before expansion. This is not essential in an industrial application of the process but, in this paper, it is important to simplify the modeling tasks. Moreover, it makes easier the comparison between the experimental and theoretical data and the validation of the mathematical model developed.

Temperature in the sample was measured by a metal sheathed thermocouple (TC Ltd., England), type T, located at its geometric center. The thermocouple, with a sheath diameter of 1 $\mathrm{mm}$, had a response time of $0.15 \mathrm{~s}$. Pressure produced in the high-pressure intensifier was monitored by a pressure transducer (0-700 MPa, EBM6045, Erich Brosa Mesgerate GmbH/KGT Kramer, Germany). All sensor measurements were recorded every $0.5 \mathrm{~s}$ by a data acquisition system (Yokogawa Data Collector 100, Tokyo, Japan).

In each experiment, a cylindrical Teflon ${ }^{\circledR}$ container (inner diameter $=28 \mathrm{~mm}$, inner height $=34 \mathrm{~mm}$, wall thickness $=0.6 \mathrm{~mm}$, capacity $=21 \mathrm{~mL}$ ) was filled with an orange juice sample. The container was closed with a screw lid, sealed by a nitrile rubber O-ring, and then it was located inside the high-pressure vessel, previously tempered at the target temperature ( -5 ${ }^{\circ} \mathrm{C}$ or $-20{ }^{\circ} \mathrm{C}$ ). A small hole at the center of the container lid, also provided with a nitrile rubber O-ring, allowed the tight insertion of the thermocouple into the sample. Then, the high-pressure vessel was closed and the pressure was risen up to the selected value and maintained until the target temperature was reached at the centre of the sample. In that moment, pressure was quickly released, in $1 \mathrm{~s}$ or less, and the sample was removed from the vessel. All the experiments were conducted, at least, in triplicate.

\subsection{Final concentration achieved in the samples after expansion}

The final concentration reached in the orange juice samples after the PSN experiments was calculated according to the procedure described by Otero et al. (2009b). Ice formation after the pressure release produces a cryoconcentration effect in the sample and, therefore, the remaining orange juice has a higher concentration, $[\mathrm{OJ}]_{\mathrm{F}}$, than the initial unfrozen sample, $[\mathrm{OJ}]_{0}$. 
The maximum temperature $\left(T_{\max }\right)$ reached in PSN experiments, after expansion and nucleation, is, by definition, the freezing point of the concentrated sample, $T_{f p}\left([O J]_{F}\right)$. Since there exists a univocal relation between the initial freezing point of a solution and its concentration, if $T_{\max }$ reached after expansion is known, the concentration of the remaining orange juice solution at this moment, $[\mathrm{OJ}]_{\mathrm{F}}$, can be easily calculated. To do that, the following semi-empirical equation, proposed by Chen et al. (1990), was employed:

$$
T_{f p}=-\frac{K_{0} X(1+C X)}{(1-X) \cdot M}
$$

where $T_{f p}$ is the freezing point of the orange juice $\left({ }^{\circ} \mathrm{C}\right), \quad K_{0}$ is the Van't Hoff's constant $\left(K_{0}=\right.$ $\left.1860{ }^{\circ} \mathrm{C} \cdot \mathrm{kg} / \mathrm{kg}-\mathrm{mol}\right), X$ is the mass fraction of solutes expressed as ${ }^{\circ} \mathrm{Brix} / 100, \mathrm{C}$ is the coefficient of solute-solvent interaction and $\mathrm{M}$ is the molecular weight of solutes. According to Chen et al. (1990), values of $C=0.25$ and $M=230$ can be used for orange juice. The validity of equation (1) was checked for the orange juice samples employed in this paper. To do that, juice samples of different concentrations $(11.8,15,20,25,30,35$ and 40 B Brix) were frozen and the corresponding freezing curves were analyzed to find the initial freezing points at the freezing plateau. At the lower concentrations (11.8 to 25 Brix), the experimental freezing points accurately agreed with those calculated from Chen's equation and a maximum deviation of 0.12 ${ }^{\circ} \mathrm{C}$ was found. Nevertheless, when increasing sample concentration, the deviation between experimental and theoretical values also increased and a maximum deviation of $0.41^{\circ} \mathrm{C}$ was found in $40{ }^{\circ}$ Brix samples. Since this maximum deviation is still quite small and it is well known that the accuracy of common methods for freezing point determination strongly decreases for highly concentrated solutions, Chen's equation was assumed to represent reasonably well the juice samples employed in this paper.

\subsection{Microscopic analysis of the ice crystals formed}

In every PSN experiment, immediately after expansion, the sample container was removed from the high-pressure vessel. Then, it was opened as quickly as possible and a small portion of the partially frozen sample was transferred to a cold microscope slide with a stainless steel micro-spoon spatula. The total time required to open the high-pressure vessel (considerably the slowest step), remove the sample container, open it and put a small portion of frozen juice at the microscope was about 2 minutes.

The ice crystals produced were observed with an Olympus BX41 microscope (Olympus, Tokyo, Japan), using transmitted light and an UPLAN FL 10X Olympus objective. To avoid thawing of the frozen samples, the microscope was fitted with a PE120 Peltier-based cooling stage coupled to a PE 94 temperature control system (Linkam Scientific Instruments, Waterfield, UK), allowing temperature control down to $-25^{\circ} \mathrm{C}$. This cooling stage and all the materials employed in the sample manipulation (spatula, coverslip glasses, tweezers and so on) were previously tempered at the freezing point of the cryoconcentrated sample $\left(T_{f p}\left([O J]_{F}\right)\right.$.

The ice crystals formed in the samples were photographed with an Olympus DP70 microscope camera (Olympus, Tokyo, Japan), interfaced to a personal computer. Then, they 
were analyzed using an image analysis software (AnalySIS FIVE, v. 5.1, Soft Imaging System $\mathrm{GmbH}$, Bensheim, Germany) which is able to detect particle boundaries and identify crystals. The spatial measurements, originally expressed as number of pixels, were calibrated by using a micrograph taken from a $1 \mathrm{~mm}$ stage micrometer placed on the microscope stage. In each photograph, not less than 100 ice crystals were identified by the program and the corresponding equivalent diameter and sphericity values were determined. The equivalent diameter was defined as the diameter of a circle with the same area than the actual area measured in the ice crystal. Sphericity describes the roundness of the ice crystal by using central moments. The sphericity of perfectly round ice crystals is equal to 1 and it decreases to 0 for more elongated crystals.

\subsection{Statistical analysis}

The results were statistically analyzed using SPSS v. 15.0 for Windows (SPSS Inc., Chicago, IL, USA). After a one-way analysis of variance (ANOVA), significant differences among means ( $\mathrm{p}$ $0.05)$ were determined by Tukey's multiple range test.

\section{RESULTS AND DISCUSSION}

\subsection{Thermal evolution in the sample during pressure-shift nucleation experiments}

Figure 2 shows a typical PSN experiment, at $200 \mathrm{MPa} /-5^{\circ} \mathrm{C}$, in a $11.8^{\circ}$ Brix orange juice sample. The juice was introduced into the high-pressure vessel, previously tempered at $-5^{\circ} \mathrm{C}$, and compressed up to $200 \mathrm{MPa}$ (COMPRESSION in Figure 2). Pressurization induced a temperature increase in the sample, from $12.80^{\circ} \mathrm{C}$ to $14.73^{\circ} \mathrm{C}$ in the experiment shown. Then, it was cooled under pressure to $-5 \stackrel{\circ}{\circ}$ (PRESSURE HOLDING STEP in Figure 2). At these conditions, the sample remained in unfrozen state according to its phase diagram (see Figure 1). Once the desired temperature was reached in the whole product, pressure was released (EXPANSION in Figure 2). This expansion induced a temperature drop in the sample, uniform in all its volume due to the isostatic nature of the pressure. Thus, the sample temperature reached a minimum value, $T_{\min }=-7.50 \stackrel{\circ}{ } \mathrm{C}$, at atmospheric conditions. This value is considerably lower than its corresponding freezing point, $\mathrm{T}_{\mathrm{fp}}\left([\mathrm{OJ}]_{0}\right)=-1.11^{\circ} \mathrm{C}$ (Chen et al., 1990), but the sample remained in a liquid metastable state, for some instants, and it did not immediately freeze. The orange juice was, therefore, highly and uniformly supercooled. After some seconds, the large supercooling reached induced a massive nucleation in the product and a certain amount of ice was formed. Figure 2 shows how the latent heat released raised the sample temperature to its new freezing point, $T_{\mathrm{fp}}\left([\mathrm{OJ}]_{\mathrm{F}}\right)$. 


\subsection{Effect of the process parameters on the duration of the pressure-shift nucleation experiments}

The pressure holding step is one of the critical steps in pressure-shift nucleation because it determines the duration of the process. The experiments made showed that the pressure and temperature conditions employed considerably affected the length of this step.

Pressure exerted a dual and opposite effect. On the one hand, it is well known that, as pressure increases, thermal conductivity is enhanced in aqueous solutions (Ramaswamy, Balasubramaniam, \& Sastry, 2007; Werner, Baars, Werner, Eder, \& Delgado, 2007) and, consequently, for a given temperature, the length of the cooling step under pressure should decrease. But, on the other hand, the higher the pressure, the larger is the work of compression and, therefore, also the subsequent rise in the sample temperature (see Table 1). As an illustration, in experiments at $-5^{\circ} \mathrm{C}$ with 11.8 ํㅏ. $\pm 0.33 \stackrel{\circ}{\circ} \mathrm{C}$ and by $9.96 \pm 0.24 \stackrel{\circ}{\circ}$ after compression up to $200 \mathrm{MPa}$ and $700 \mathrm{MPa}$, respectively. For the more concentrated samples, the temperature rise was even higher and, in 40 Brix orange juices, temperature increases in the aforementioned experiments were $4.54 \pm 0.13 \stackrel{\circ}{\circ} \mathrm{C}$ and $13.71 \pm 0.04 \stackrel{\circ}{\circ}$. These rises in the sample temperature after compression should extend the length of the cooling step under pressure. In the experiments made at the lower pressures, the enhanced thermal conductivity accelerated the removal of the heat generated during the compression and counteracted its effect but, at the higher pressures, the effect of compression was predominant. For example, $49.6 \pm 0.7$ min were needed to reach $-5 \stackrel{\circ}{ } \mathrm{C}$ in experiments at $700 \mathrm{MPa}$ with $40 \stackrel{\circ}{\circ}$ Brix orange juice samples while only $31.3 \pm 0.4$ min were needed in experiments at $200 \mathrm{MPa}$. This difference, relatively small in lab-scale experiments, could be important in an industrial process with large volumes of product which make heat extraction more difficult.

Temperature also affected the length of the cooling step under pressure. Thus, in the experiments made at $-20 \stackrel{\circ}{\circ} \mathrm{C}$, heat removal from the system was more efficient and, consequently, the temperature increases recorded after compression were lower in all the cases (see Table 1). Nevertheless, more sensible heat must be removed from the sample to reach the target temperature and, therefore, PSN experiments made at $-20 \stackrel{\circ}{ } \mathrm{C}$ were always longer than those performed at $-5 \stackrel{\circ}{\circ}$.

Juice concentration did not significantly affect the duration of the pressure holding step. As previously commented, the temperature rise after compression was higher in the more concentrated samples, but these samples were cooled more quickly due to their lower heat capacity. It is important to stress that the samples employed in this paper were relatively small and, therefore, no important effects due to differences in viscosity were observed. Nevertheless, the higher viscosity of the more concentrated samples could hamper mass transfer and heat removal in an industrial process.

It must be emphasized that the very long cooling times recorded in the small samples employed in this paper are not representative of the cooling times expected in an industrial application of pressure-shift nucleation. The long times recorded in the experiments are the 
direct consequence of the especial conditions at which they were performed. On the one hand, as previously commented, experiments in this paper were mainly designed to prove the validity of the model presented. Therefore, the cooling system was maintained at only $0.5^{\circ} \mathrm{C}$ under the target temperature to minimize thermal gradients in the sample before expansion and, in this way, simplify the validation. On the other hand, the experiments were performed at a lab-scale high-pressure equipment, not optimized to accelerate heat removal from the sample. Moreover, the sample holder was built in Teflon $\AA$, a good thermal insulator, and the pressure medium was silicon oil which undergoes high temperature increases after compression. All these factors, which negatively affected the heat removal from the sample and induced long cooling times under pressure, can be optimized when designing an industrial process.

\subsection{Effect of the process parameters on the supercooling extent and the nucleation rate reached after expansion}

Once the target temperature was reached in the sample, pressure was quickly released (see Figure 2). Expansion induced a temperature drop in the juice and it remained in a supercooled state for some instants. The extent of supercooling $(\Delta T)$ is a determinant factor in pressure-shift nucleation since it is well known that the nucleation rate and, therefore, the amount of ice quasi-instantaneously formed, strongly increase when it rises (Heneghan, Wilson, \& Haymet, 2002; Kiani \& Sun, 2011; Petzold \& Aguilera, 2009; Reid, 1983).

In this paper, $\Delta T$ was calculated as the difference between the initial freezing point of the sample, $T_{\mathrm{fp}}\left([\mathrm{OJ}]_{0}\right)$, and the minimum temperature reached after expansion, $T_{\min }$, just before nucleation. The obtained results are shown in Table 2. It is important to note that, in the experiments made, sensor measurements were recorded every $0.5 \mathrm{~s}$. Therefore, when nucleation took place very quickly, in $1 \mathrm{~s}$ or less after expansion, only 1 or 2 temperature measurements could be carried out to detect $T_{\min }$ and $\Delta T$. This is not enough to ensure that the $T_{\min }$ value recorded was not masked, in a certain extent, by the release of latent heat when nucleation occurred. In these cases, the real $\Delta T$ determination was not feasible and an asterisk appears in Table 2.

Table 2 clearly shows the effect of the pressure conditions employed in the PSN experiments on the extent of supercooling reached after expansion. The higher the pressure, the larger was the temperature drop provoked and, hence, the supercooling induced in the sample. The large $\Delta T$ reached in the experiments at the higher pressures induced extremely fast nucleations, especially in the less concentrated samples and, as previously commented, real $\mathrm{T}_{\min }$ and $\Delta \mathrm{T}$ values could not be recorded. These results confirm previous findings made by a number of authors (Chevalier, Le Bail, \& Ghoul, 2000; Otero \& Sanz, 2006; Otero et al., 2009b) who had already described an increase in the extent of supercooling reached in different samples when increasing pressure before expansion.

The effect of the temperature conditions employed in the experiments is depicted in Figure 3. It shows some representative experiments at $350 \mathrm{MPa}$ and two different temperatures, $-5 \stackrel{\circ}{\circ}$ and $-20{ }^{\circ} \mathrm{C}$, in $11.8 \stackrel{\circ}{\circ}$ Brix and $40 \stackrel{\circ}{\circ}$ Brix orange juice samples. In experiments at $-5{ }^{\circ} \mathrm{C}$ 
(Figures $3 a$ and $3 b$ ), $\Delta T$ was relatively low and nucleation took place in the sample at atmospheric pressure, after remaining some seconds in a supercooled state. When temperature was lowered to $-20 \stackrel{\circ}{ } \mathrm{C}$, supercooling was considerably increased and, therefore, very quick nucleations were induced either under pressure (Figure 3c) or at atmospheric conditions (Figure $3 d)$. This enhancement of supercooling when lowering temperature before expansion had been already described by Otero et al. (2006; 2009b).

Figure 3 shows how the initial juice concentration also had an influence on the extent of supercooling reached. The more concentrated the juice, the lower was the extent of supercooling reached due to the freezing point depression experimented (compare ice melting curves and $\Delta T$ in Figures $3 a$ and $3 b$ ). Similar results were reported by Thiebaud et al. (2002) who found a decrease in the extent of supercooling reached in oil in water emulsions after expansion from $200 \mathrm{MPa} /-18{ }^{\circ} \mathrm{C}$ when increasing their fructose concentration between 0 and $25 \%$.

Figure 4 shows in detail the temperature evolution in orange juice samples just after expansion from different pressure and temperature conditions in representative PSN experiments. The figure illustrates the temperature drop induced in the samples by the pressure release and the subsequent nucleation, which can be easily identified by a sudden rise in the temperature curves. Note that, in experiments at $200 \mathrm{MPa} /-5{ }^{\circ} \mathrm{C}$, the extent of supercooling reached in $40{ }^{\circ}$ Brix samples, $\Delta \mathrm{T}=3.46 \pm 0.17{ }^{\circ} \mathrm{C}$, was not enough to induce nucleation. Therefore, these samples could not be frozen at these conditions and the slight temperature increase observed in Figure 4a was only due to the thermal exchange between the sample and the HP vessel tempered at $-5 \stackrel{\circ}{\circ}$.

It is well known that ice nucleation is a stochastic phenomenon (Heneghan et al., 2002; Kiani \& Sun, 2011; Wilson \& Haymet, 2009), but many factors can affect the probability of nucleation including the extent of supercooling, the rate of cooling, the volume of the sample, its concentration or the type of container in which it is located, among others (Kiani \& Sun, 2011; Wolfe \& Bryant, 1999). Lag times observed in Figure 4 between the pressure release and ice nucleation clearly show the effects of the extent of supercooling and the sample concentration on the probability of nucleation. Thus, for a given sample, the larger the observed $\Delta \mathrm{T}$, the earlier nucleation occurred. See, for example, 20 Brix samples: nucleation occurred at about $14 \mathrm{~s}$ (Figure 4a) and $5 \mathrm{~s}$ (Figure $4 \mathrm{~b}$ ) after expansion in the experiments shown at $200 \mathrm{MPa} /-5^{\circ} \mathrm{C}(\Delta \mathrm{T}$ $\left.=6.05{ }^{\circ} \mathrm{C}\right)$ and $350 \mathrm{MPa} /-5^{\circ} \mathrm{C}\left(\Delta \mathrm{T}=9.28^{\circ} \mathrm{C}\right)$, respectively. In experiments at $-20{ }^{\circ} \mathrm{C}$, observed $\Delta \mathrm{T}$ values were considerably larger, although accurate values could not be measured, and nucleation occurred much more quickly: $0.5 \mathrm{~s}$ after the pressure release (Figure $4 \mathrm{c}$ ) in the experiment at $200 \mathrm{MPa}$ or even during expansion in the experiment at $350 \mathrm{MPa}$ (Figure 4d).

For a given treatment, nucleation occurred earlier and faster in the less concentrated juices because they reached higher supercooling extents (see Table 2). Moreover, an ice nucleus can only be formed if, at a particular time, a volume greater or equal to the critical volume is free of solute molecules (Wolfe \& Bryant, 1999). Therefore, the physical presence of many solute molecules in the more concentrated solutions hinders the formation of nuclei. In 
addition, solutes also increase the sample viscosity at a given temperature and this also makes the motion and reorientation of the water molecules into the ice structure more difficult (Petzold \& Aguilera, 2009; Wolfe \& Bryant, 1999). Therefore, in the more concentrated samples, the probability of nucleation considerably decreased and it took place slowly as the slope of the curves in Figure 4 illustrates. These results agree with those obtained by Thiebaud et al. (2002) and Otero et al. (2009b) in oil in water emulsions and sucrose solutions, respectively. All these authors described a considerable decrease in the rate at which temperature increased after nucleation when increasing the sugar concentration of the sample.

After nucleation, temperature increased up to a maximum value, $T_{\max }$, due to the latent heat released by the ice formed. Ice formation produced a cryoconcentration effect in the sample and, therefore, $T_{\max }$ is the freezing point of the remaining concentrated solution, $\mathrm{T}_{\mathrm{fp}}\left([\mathrm{OJ}]_{\mathrm{F}}\right)$.

\subsection{Final concentration achieved in the samples after expansion}

Table 3 shows the mean $T_{\max }$ values recorded in the orange juice samples after expansion from different pressure/temperature conditions. As commented in section 3.3, 40 Brix orange juice samples did not freeze when they were processed at $200 \mathrm{MPa} /-5 \stackrel{\circ}{\mathrm{C}}$ and, therefore, no $T_{\max }$ value is presented for these conditions. The standard deviations found, usually lower than $0.1 \stackrel{\circ}{\circ}$, indicate the good repeatability of the measurements. Table 3 shows

how, for a given sample, $T_{\max }$ decreased when pressure before expansion increased and/or temperature decreased; in other words, $\mathrm{T}_{\max }$ decreased when the extent of supercooling increased.

From these $T_{\max }$ values, the final concentration achieved in each sample after nucleation was calculated using equation (1). Figure 5 clearly illustrates how, for a given sample, the higher the pressure and/or the lower the temperature before expansion, the larger was the final concentration attained. At these conditions, large extents of supercooling were reached which induced a quick and massive ice nucleation. In $20{ }^{\circ}$ Brix samples, for example, concentration was increased up to $21.82 \pm 0.25,23.23 \pm 0.43,23.78 \pm 0.16$ and $24.53 \pm 0.33$ BBrix, after expansions from $-5 \stackrel{\circ}{\circ}$ and $200,350,500$ and $700 \mathrm{MPa}$, respectively. Higher concentration levels were, however, achieved in experiments at $-20{ }^{\circ} \mathrm{C}: 25.96 \pm 0.53$ and 26.78 \pm 0.44 B Brix, after expansions from only 200 and $350 \mathrm{MPa}$. Lowering temperature before expansion is, therefore, a more effective way to obtain large [OJ] $]_{F}$ than increasing pressure, but economic implications should be also taken into account when designing the corresponding industrial process.

\subsection{Mathematical model to predict the final concentration attained in orange juice samples after pressure-shift nucleation}

The final concentration attained in the samples after pressure-shift nucleation, $[\mathrm{OJ}]_{F}$ in -Brix, can be calculated from the pressure and temperature conditions employed in the process, $\mathrm{P}(\mathrm{MPa})$ and $\mathrm{T}\left({ }^{\circ} \mathrm{C}\right)$, and the initial concentration of the orange juice sample, $[\mathrm{OJ}]_{0}$ in ${ }^{\circ}$ Brix. 
The initial concentration of the sample can be expressed as:

$$
\mathrm{PJ}_{\underline{0}}^{-}=\frac{\mathrm{m}_{\mathrm{ss}}}{\mathrm{m}_{\mathrm{w}}^{0}+\mathrm{m}_{\mathrm{ss}}} \cdot 100
$$

where $\mathrm{m}_{\mathrm{w}}^{0}$ and $\mathrm{m}_{\mathrm{ss}}$ are the mass fractions of water and soluble solids in the juice. It is obvious that before crystallization:

$$
m_{\mathrm{w}}^{0}+\mathrm{m}_{\mathrm{ss}}=1
$$

and

$$
\mathrm{m}_{\mathrm{ss}}=\text { Cons tant }
$$

After crystallization,

$$
\mathrm{m}_{\mathrm{w}}^{\mathrm{F}}+\mathrm{m}_{\mathrm{i}}+\mathrm{m}_{\mathrm{ss}}=1
$$

where $m_{w}^{F}$ and $m_{i}$ are the mass fractions of remaining liquid water and ice, respectively.

The final concentration in the juice, after the pressure release, can be deduced from equations (2-5):

$$
D J_{E}^{-}=\frac{m_{s s}}{m_{w}^{F}+m_{s s}} \cdot 100=\frac{D_{\underline{o}}^{-}}{1-m_{i}}
$$

This equation is only valid if retention of solids in the ice formed is negligible (Deshpande, Bolin, \& Salunkhe, 1982). In other case:

$$
P J_{E}^{-}=\frac{m_{s s}-\alpha \cdot m_{i}}{m_{w}^{F}+\left(m_{s s}-\alpha \cdot m_{i}\right)} \cdot 100
$$

where $\alpha$ is the concentration of soluble solids removed in the ice formed (g solids/g ice).

In this paper, the soluble solids retention in the ice formed has been considered, as a first approximation, negligible. Therefore, $m_{i}$ is the only unknown variable to solve equation (6). According to Otero and Sanz $(2000,2006), m_{i}$ can be predicted from this simple heat balance:

$$
\overline{\mathrm{cp}_{\mathrm{w}}} \cdot \Delta \mathrm{T} \cdot \mathrm{m}_{\mathrm{w}}^{\mathrm{F}}+\overline{\mathrm{cp}_{\mathrm{i}}} \cdot \Delta \mathrm{T} \cdot \mathrm{m}_{\mathrm{i}}+\overline{\mathrm{cp}_{\mathrm{dm}}} \cdot \Delta \mathrm{T} \cdot \mathrm{m}_{\mathrm{dm}}=\mathrm{m}_{\mathrm{i}} \cdot \mathrm{L}_{\mathrm{w}}
$$

where $\overline{\mathrm{cp}_{\mathrm{w}}}, \overline{\mathrm{cp}_{\mathrm{i}}}$ and $\overline{\mathrm{cp}_{\mathrm{dm}}}$ are the specific heat capacities of water, ice and dry matter (mean value of $c p$ at $T_{\min }$ and at $T_{f p}\left([O J]_{0}\right)$, both at atmospheric conditions), $L_{w}$ is the latent heat of pure water at atmospheric pressure and $m_{d m}$ is the mass fraction of dry matter in the sample. Since the orange juice concentrate employed to prepare the samples was obtained from a clarified juice, it can be assumed that $\mathrm{m}_{\mathrm{ss}} \cong \mathrm{m}_{\mathrm{dm}}$. In this paper, $\overline{\mathrm{cp}_{\mathrm{w}}}$ and $\overline{\mathrm{cp}_{\mathrm{i}}}$ were obtained, as a function of temperature, from the calculation routines published by Otero et al. (2002). Moreover, $\overline{\mathrm{Cp}_{\mathrm{dm}}}$ was obtained from:

$$
\overline{c p_{d m}}=\left(c p_{p} \cdot m_{p}+c p_{f} \cdot m_{f}+c p_{c} \cdot m_{c}+c p_{f i b} \cdot m_{f i b}+c p_{a} \cdot m_{a}\right) / m_{d m}
$$


where $m_{p}, m_{f}, m_{c}, m_{\text {fib }}$ and $m_{a}$ are the mass fractions of protein, fat, carbohydrates, fiber and ash in the orange juice samples. Composition data from the Danish Food Composition Databank (Saxholt et al., 2008) were employed to make these calculations. Specific heat values for protein $\left(\mathrm{cp}_{\mathrm{p}}\right)$, fat $\left(\mathrm{cp}_{\mathrm{f}}\right)$, carbohydrates $\left(\mathrm{cp}_{\mathrm{c}}\right)$, fiber $\left(\mathrm{cp}_{\mathrm{fib}}\right)$ and ash $\left(\mathrm{cp}_{\mathrm{a}}\right)$ were obtained, as a function of temperature, from the equations proposed by Choi and Okos (1986).

Equation (8) can be applied if nucleation occurs at atmospheric conditions and its solution involves a correct $\Delta T$ prediction. But, as previously commented, nucleation, which is a stochastic process, can take place under pressure during expansion (see, as an example, Figure $3 \mathrm{c}$ ) and; therefore, $\Delta \mathrm{T}$ prediction is a problem when modeling. Nevertheless, Otero and Sanz (2006) proved that, for modeling purposes, nucleation may be considered to always occur at atmospheric pressure if expansions are quickly performed. Then, $\Delta T$ can be calculated from:

$$
\Delta \mathrm{T}=\mathrm{T}_{\mathrm{fp}} \mathrm{PJ}_{\underline{0}}^{-}-\mathrm{T}_{\min }
$$

The minimum temperature reached in the sample after expansion can be estimated using Equation (11) if the thermophysical properties of the sample, under pressure, are known:

$$
\left(\frac{\partial T_{k}}{\partial p}\right)_{S}=\frac{\alpha \cdot V}{c p} \cdot T_{k}
$$

where $T_{k}$ is the temperature $(K), p$ represents pressure $(\mathrm{Pa}), \alpha$ is the thermal expansion coefficient $\left(\mathrm{K}^{-1}\right)$ and $\mathrm{V}$ is the specific volume $\left(\mathrm{m}^{3} / \mathrm{kg}\right)$. Unfortunately, these properties were not available in the literature for orange juices. Therefore, in this paper, thermophysical properties of pure water (Otero et al., 2002) were employed to calculate $T_{\min }$ as an approximation.

Figure 6 compares $[\mathrm{OJ}]_{F}$ predictions for orange juices samples, expanded from different pressure and temperature conditions, with $[\mathrm{OJ}]_{F}$ values calculated from the experimental $T_{\max }$ data (mean \pm standard deviation values). Predictions for pressure-shift nucleations from 700 $\mathrm{MPa} /-5^{\circ} \mathrm{C}$ could not be made because $\mathrm{V}, \alpha$ and $\mathrm{cp}$ values, needed to solve equation (11), were not available in the literature for liquid water at these conditions (metastable state). Figure 6 reveals the good agreement between the model predictions and the experimental data. Thus, the maximum difference, in absolute value, between both sets of data was 1.8 "Brix. These results can be considered accurate enough taking into account the approximations assumed in the predictions.

\subsection{Ice crystals formed after pressure-shift nucleation}

In every PSN experiment, immediately after expansion, the sample container was taken out of the pressure vessel and opened. Expansion induced a uniform supercooling throughout the sample, due to the isostatic nature of the pressure, and it promoted the instantaneous crystallization of a relatively large percentage of water in the whole sample. Thus, in all the

experiments made, the ice crystals observed after opening the sample container were 
homogeneously distributed in the whole volume of the juice and not only at the container surface as occurs in conventional freezing.

Figure 7 shows some of the microphotographs taken in $11.8{ }^{\circ}$ Brix orange juice samples after expansion in different PSN experiments. Pressure and temperature conditions employed in the experiments strongly affected the number and size of the ice crystals formed. In the experiments made at the higher pressures and/or the lower temperatures, much more ice crystals were formed (see, for example, the photograph corresponding to $200 \mathrm{MPa} /-5 \stackrel{\circ}{\circ} \mathrm{C}$ and compare to the photograph corresponding to $\left.700 \mathrm{MPa} /-5{ }^{\circ} \mathrm{C}\right)$. This finding agrees with observations made by Fuchigami et al. (2002) who reported an increasing number of ice crystals in tofu samples when increasing pressure at the expansion from $200 \mathrm{MPa}$ to $600 \mathrm{MPa}$. Figure 7 also shows how the ice crystals were considerably smaller in experiments made at the higher pressures and/or the lower temperatures. These rough observations were corroborated when the equivalent diameter of the ice crystals was measured. Thus, the statistical analysis made revealed that both pressure and temperature significantly affected $(p<0.05)$ the mean equivalent diameter of the crystals formed. As an illustration, Figure 8 compares some typical ice crystal size distributions obtained after expansion from different pressure and temperature conditions in 11.8 "Brix orange juice samples. As pressure increased and/or temperature decreased or, in other words, as the extent of supercooling reached after expansion increased, the size distributions narrowed and most of the ice crystals were located in class intervals of lower size. In particular, $15.8 \%$ ice crystals had an equivalent diameter smaller than $40 \mu \mathrm{m}$ after expansions from $200 \mathrm{MPa} /-5{ }^{\circ} \mathrm{C}$. This percentage rose to $53.6 \%$ or to $54.5 \%$ when pressure increased up to $700 \mathrm{MPa}$ or temperature decreased to $-20 \stackrel{\circ}{\circ} \mathrm{C}$, respectively. These results corroborate previous findings by a number of authors who also showed that higher pressures and/or lower temperatures at the expansion produced significantly higher extents of supercooling and, consequently, smaller ice crystals in different high-pressure shift frozen products (Chevalier et al., 2000; Fernández, Martino, Zaritzky, Guignon, \& Sanz, 2007; Fuchigami et al., 2002; Kulisiewicz, Kowalczyk, Baars, \& Delgado, 2007; Zhu, Le Bail, \& Ramaswamy, 2003; Zhu, Le Bail, Ramaswamy, \& Chapleau, 2004; Zhu, Ramaswamy, \& Le Bail, 2005).

Figure 7 also shows how the ice crystals formed in PSN experiments were relatively round, with smooth edges in all the cases and no dendritic shapes were found. Mean sphericity values ranged from 0.40 to 0.55 in the samples analyzed and no strong effect of pressure and temperature conditions on the roundness was found. Only crystals formed after expansion from $200 \mathrm{MPa} /-5 \stackrel{\circ}{\circ} \mathrm{C}$ were significantly $(p<0.05)$ less round than the rest. Similar results were found by Zhu et al. $(2004 ; 2005)$ who did not find significant differences between the roundness of ice crystals formed in gelatin and pork muscle samples high-pressure shift frozen at different pressure levels, between 100 and $200 \mathrm{MPa}$.

The initial concentration of the juice samples also affected the characteristics of the ice crystals formed. Precise comparisons between all the samples could not be made because the most concentrated juices, 40 Brix samples, were partially thawed during the sample 
manipulation. The photographs made revealed that the number of ice crystals formed after expansion was lower in the more concentrated samples, presumably because the extent of supercooling reached in these samples was also lower. As an example, in PSN experiments at $350 \mathrm{MPa} /-5 \stackrel{\circ}{\circ}$, the number of ice crystals counted in the photographs of $11.8,20$ and 30 B. Brix samples was $239 \pm 20,171 \pm 33$ and $123 \pm 11$, respectively.

\subsection{Implementation of pressure-shift nucleation in a freeze concentration process}

In the experiments made, final concentrations attained in 11.8 'Brix samples were comprised between $13.02 \pm 0.00$ and $18.44 \pm 0.91$ Brix, depending on the pressure and temperature conditions assayed. These values are considerably lower than those obtained by traditional concentration methods. In fact, 70-75 ${ }^{\circ}$ Brix, 30-40 ${ }^{\circ}$ Brix or 45 Brix can be achieved in clarified juices after concentration by heat evaporation, membrane concentration or freeze concentration, respectively. It is obvious; therefore, that pressure-shift nucleation cannot be employed as a one-step process to cryoconcentrate fresh single-strength juices up to those levels. Moreover, increasing concentration from $11.8 \stackrel{\circ}{\circ}$ Brix to $45^{\circ}$ Brix in one step would involve to separate $73.8 \%$ initial juice mass as ice crystals and this would be a really difficult task.

However, Figure 5 shows that PSN experiments in $40{ }^{\circ}$ Brix samples produced [OJ] $]_{F}$ values between $43.84 \pm 0.14$ and $49.57 \pm 0.20 \stackrel{\circ}{ }$ Brix, values close to those obtained in traditional freeze concentration. Therefore, multistage processes could be designed to progressively reach the level of concentration desired after several expansions. Nevertheless, this design implies high capital costs to acquire several pressure vessels. Another alternative, shown in Figure 9, would be to adjust the initial concentration of the sample (by mixing part of the final product with the fresh single-strength juice) at the level needed to reach the desired final concentration just after one expansion from the pressure and temperature conditions chosen (Otero, Sanz, \& Guignon, 2009a). Thus, to obtain juice concentrates with a final concentration of 45 Brix, for example, the initial concentration of the sample, at the steady regime, should be close to $40{ }^{\circ}$ Brix. The exact [OJ] $]_{0}$ value depends on the pressure and temperature conditions chosen for the PSN process and it can be calculated using the mathematical model developed. Thus, as an illustration, the initial concentration of the juice should be adjusted to $41.6{ }^{\circ} \mathrm{Brix}, 38.5^{\circ} \mathrm{Brix}$ or $35.2{ }^{\circ} \mathrm{Brix}$ when the process conditions are 300 $\mathrm{MPa} /-5 \stackrel{\circ}{\circ} \mathrm{C}, 700 \mathrm{MPa} /-5{ }^{\circ} \mathrm{C}$ or $350 \mathrm{MPa} /-20 \stackrel{\circ}{ } \mathrm{C}$, respectively. To achieve these [OJ] values, a part of the final concentrate obtained must be re-circulated to the high-pressure vessel and be mixed with fresh single-strength juice (see Figure 9). The juice is then introduced in the highpressure vessel and cooled under pressure. Once the target temperature is reached in the sample, an expansion is made. Ice crystals formed and the concentrated solution go together to the recrystallization tank. Here, the concentrated solution is separated and the ice crystals grow to reach a size large enough to be easily washed at the wash column. In this way, [OJ] $]_{F}$ can be efficiently controlled by adjusting the initial concentration of the sample and the pressure and temperature conditions of the process (Otero et al., 2009a). 


\subsection{Industrial relevance and feasibility of the process}

The results obtained in this paper show that pressure-shift nucleation presents a number of advantages over the traditional crystallization step at the scraped surface heat exchanger which prove the industrial relevance of the process proposed.

First of all, it is interesting to note that, in traditional crystallization, temperature at the scraped surface heat exchanger must be set always lower than the temperature at which nucleation occurs in the sample. Moreover, temperature must be low enough to ensure high flow rates and low residence times at the SSHE to make the process efficient. Pressure-shift nucleation makes possible to work at temperatures considerably higher than those employed in traditional crystallization, even higher than $\mathrm{T}_{\mathrm{fp}}\left([\mathrm{OJ}]_{0}\right)$. As an illustration, the freezing point of 40 Brix orange juice is $-5.93 \stackrel{\circ}{ } \mathrm{C}$ (Chen et al., 1990), but it can be pressure-shift nucleated at $-5 \stackrel{\circ}{ }{ }^{\circ}$ if pressure is high enough (see Figure 5). Pressure release induces a temperature drop in the sample which is higher when pressure increases. In 40 -Brix samples, the minimum temperatures reached after expansion were $-9.39 \pm 0.17 \stackrel{\circ}{\circ} \mathrm{C},-12.82 \pm 0.00 \stackrel{\circ}{ } \mathrm{C},-16.09 \pm 0.15 \stackrel{\circ}{ } \mathrm{C}$ and $-19.63 \pm 0.05^{\circ} \mathrm{C}$ in experiments at $200 \mathrm{MPa} /-5 \stackrel{\circ}{\circ} \mathrm{C}, 350 \mathrm{MPa} /-5{ }^{\circ} \mathrm{C}, 500 \mathrm{MPa} /-5{ }^{\circ} \mathrm{C}$ and 700 $\mathrm{MPa} /-5 \stackrel{\circ}{\circ} \mathrm{C}$, respectively. Therefore, the extent of supercooling was $3.63 \pm 0.14 \stackrel{\circ}{ } \mathrm{C}, 6.89 \pm 0.00$ ${ }^{\circ} \mathrm{C}, 10.16 \pm 0.15 \stackrel{\circ}{\circ} \mathrm{C}$ and $13.7 \pm 0.05{ }^{\circ} \mathrm{C}$ in each case. The results obtained showed that the supercooling reached at $200 \mathrm{MPa} /-5 \stackrel{\circ}{\circ} \mathrm{C}$ was not enough to induce ice nucleation in $40{ }^{\circ}$ Brix samples, but final concentrations of $43.84 \pm 0.14{ }^{\circ}$ Brix, $45.46 \pm 0.32{ }^{\circ}$ Brix and $46.47 \pm 0.17{ }^{\circ}$ Brix were achieved after expansions from $350 \mathrm{MPa} /-5 \stackrel{\circ}{\circ} \mathrm{C}, 500 \mathrm{MPa} /-5 \stackrel{\circ}{\circ} \mathrm{C}$ and $700 \mathrm{MPa} /-5 \stackrel{\circ}{\circ} \mathrm{C}$, respectively. This is an important advantage over the traditional process in that a substantial part of the economic cost of freeze concentration is due to the low temperatures needed.

Second, in traditional crystallization, ice nucleation only occurs at the refrigerated walls of the scraped surface heat exchanger. Therefore, mechanical scrapers, in continuous rotation, are necessary to remove ice crystals from the SSHE walls. In pressure-shift nucleation, ice is formed in the whole volume of the sample at the same time and not only at the surface in direct contact with the refrigerated walls. After expansion, a sort of semi-fluid ice slurry is obtained and there is no need of continuous scraping to remove ice crystals from the walls.

Third, in traditional crystallization, the product must be continuously circulating between the scraped surface heat exchanger and the re-crystallization tank to progressively increase the amount of ice formed and reach, in this way, the desired concentration level. In the PSN process proposed, the final concentration is reached in the whole volume of the sample quasiinstantaneously, just after expansion. Moreover, final concentration can be finely adjusted by choosing the appropriate initial juice concentration and pressure/ temperature conditions.

Fourth, in any freeze concentration process, the size and shape of the ice crystals formed are parameters of utmost importance because they determine the cleanness of the separation process. The velocity at which stable, clean displacement can be obtained in wash columns is proportional to the ice bed permeability. This parameter is markedly reduced by relatively small amounts of small particles. Therefore, to obtain clean separations, ice-concentrate slurries should not only have a large average crystal size, but they also should contain as few small 
crystals as possible (Smith \& Schwartzberg, 1985). Moreover, the ice crystals should be convex and not contain pockets or indentations in which the concentrated solution might be trapped. In traditional freeze concentration, ice crystals formed in the scraped-surface heat exchanger are dendritic and have an equivalent diameter between 1 and $10 \mu \mathrm{m}$ (Van Nistelrooij, 2005). As commented in the Introduction section, these crystals are not appropriate to be efficiently separated at the wash column and, therefore, they must be sent to a ripening tank where they make their edges round and increase their size up to 300-500 $\mu \mathrm{m}$. Ice crystals formed after expansion in the PSN experiments were considerably larger and round for all the pressure and temperature conditions assayed.

Concerning the feasibility of the process, it is evident that the implementation of pressureshift nucleation as a substitute of the crystallization step at the scraped surface heat exchanger involves the previous development of high-pressure equipment especially adapted to the process. Current high-pressure machines in the food industry are not appropriate for the process proposed because they are not designed to work at sub-zero temperatures and the vessels are too wide to allow a quick heat removal from the product. The equipment to employ in PSN should be able to quickly cool large amounts of product under pressure in a semicontinuous mode. High-pressure tubular reactors, like those well-established in the chemical industry for the generation of low density polyethylene (LDPE), could be an appropriate solution. These reactors consist of several tubes connected together with $180^{\circ}$ bends which can reach a total length of $3000 \mathrm{~m}$. High-pressure tubular reactors in a LDPE plant operate at pressures about $300 \mathrm{MPa}$ and temperatures close to $300^{\circ} \mathrm{C}$ and they can render an annual output of 400000 tons (Asteasuain \& Brandolin, 2009; Kiparissides et al., 2005; Trieb, Karl, \& Moderer, 2007). This concept of tubular reactor, especially adapted for high-pressure shift freezing, has been already explored by Otero et al. (2007) and Urrutia et al. (2007). A high-pressure lowtemperature tubular reactor would consist of a processing pipe, continuously subjected to high pressure and ended with pressure-balanced ball valves which allow a semi-continuous process. The processing pipe would be surrounded by a cooling jacket to quickly reach the target temperature desired. As an example, Otero et al. (2007) calculated the length needed in a tubular reactor (60 $\mathrm{mm}$ inner diameter and $129 \mathrm{~mm}$ outer diameter) to freeze tylose samples at $260 \mathrm{MPa} /-26{ }^{\circ} \mathrm{C}$ when the sample velocity ranged from 1 to $6 \mathrm{~m} / \mathrm{min}$. The vessel lengths obtained were shorter than $300 \mathrm{~m}$ in all the cases, a value significantly lower than the usual lengths of LDPE reactors. Therefore, important annual outputs could be achieved if this kind of reactor were employed for freeze concentration. These results encourage to exploit the advantages of pressure-shift nucleation in the food industry, but more research work on this kind of high-pressure reactors, able to work in a semi-continuous mode, is needed.

\section{CONCLUSIONS}

In this paper, pressure-shift nucleation has been evaluated as a potential tool to improve suspension cryoconcentration processes. The results obtained show that PSN could be 
employed as a substitute of the crystallization step at the scraped surface heat exchanger in traditional freeze concentration.

Pressure-shift nucleation presents four important advantages over the conventional crystallization. First, temperature at the high-pressure vessel can be set considerably higher than at the scraped surface heat exchanger and this can involve an important energetic saving. Second, in PSN, ice crystals are instantaneously produced in the whole volume of the sample and not only at the surface close to the heat exchanger. Therefore, no complex scraping devices are needed. Third, in PSN, the final concentration is achieved in the whole sample just after expansion. Therefore, there is no need to recirculate the product to progressively increase the amount of ice formed to reach the desired concentration. Moreover, the final concentration can be efficiently controlled by fitting the initial juice concentration in the high-pressure vessel and the pressure and temperature conditions employed in the process. Fourth, the ice crystals formed after expansion are round, without pockets and indentations.

The adequate fitting of all the aforementioned process parameters is crucial because it will determine the economic viability of the cryoconcentration process. When choosing the optimal conditions, it is important to consider not only the conditions which maximize the production rate, but also those which minimize the costs: compromise solutions should be adopted. The results obtained in this paper show that the higher the pressure and the lower the temperature, the larger is the final concentration attained in the sample, but also the smaller are the ice crystals formed. These pressure and temperature conditions involve the higher economic costs, not only due to the longer processing times needed (both at the high-pressure vessel and at the recrystallizer) but also due to the refrigeration power implied. It is interesting to note that increasing pressure at relatively high temperatures could reduce costs and, also, microbial and/or enzymatic inactivation could be produced as an added value in the cryoconcentration process. This can be considered a fifth important advantage of PSN over traditional crystallization, especially in thermolabile food products which need a stabilization process.

All the advantages described indicate that pressure-shift nucleation could be an interesting substitute of the crystallization step at the scraped surface heat exchanger but, before implanting the system at the food industry, it is necessary to develop high-pressure equipments especially adapted to the process proposed.

\section{ACKNOWLEDGEMENTS}

This work has been supported by the National Plan of Spanish $1+D+i$ of the Ministry of Education and Science (MEC), through the projects AGL2007-63314/ALI and CSD2007-00045 MALTA CONSOLIDER-INGENIO 2010, the Madrid Community through the project QUIMAPRES S2009/PPQ-1551 and the research group MOMAT (Ref. 910480) funded by Banco Santander and Universidad Complutense de Madrid. B. Guignon has a contract from CSIC (JAE program). 


\section{REFERENCES}

Aider, M., \& de Halleux, D. (2009). Cryoconcentration technology in the bio-food industry: Principles and applications. LWT - Food Science and Technology, 42(3), 679-685.

Asteasuain, M., \& Brandolin, A. (2009). High-pressure polymerization of ethylene in tubular reactors: A rigorous dynamic model able to predict the full molecular weight distribution. Macromolecular Reaction Engineering, 3(7), 398-411.

Botsaris, G., \& Qian, R. (1999). Process and system for freeze concentration using ultrasonic nucleation useful in effluent processing. United States Patent. Number: 5966966. October 19.

Braddock, R. J., \& Marcy, J. E. (1985). Freeze concentration of pineapple juice. Journal of Food Science, 50(6), 1636-1639.

Cheftel, J. C., Lévy, J., \& Dumay, E. M. (2000). Pressure-assisted freezing and thawing: Principles and potential applications. Food Reviews International, 16(4), 453483.

Chen, C. S., Nguyen, T. K., \& Braddock, R. J. (1990). Relationship between freezing point depression and solute composition of fruit juice systems. Journal of Food Science, 55(2), 566-567.

Chevalier, D., Le Bail, A., \& Ghoul, M. (2000). Freezing and ice crystals formed in a cylindrical food model: part II. Comparison between freezing at atmospheric pressure and pressure-shift freezing. Journal of Food Engineering, 46(4), 287293.

Choi, Y., \& Okos, M. R. (1986). Effects of temperature and composition on the thermal properties of food. In M. L. Maguer \& P. Jelen (Eds.), Food Engineering and Process Applications. Vol. 1. Transport phenomena (pp. 93-101). London: Elsevier Applied Science.

Deshpande, S. S., Bolin, H. R., \& Salunkhe, D. K. (1982). Freeze concentration of fruit juices. Food Technology, 36(May), 68-82.

Deshpande, S. S., Cheryan, M., Sathe, S. K., Salunkhe, D. K., \& Luh, B. S. (1984). Freeze concentration of fruit juices. CRC Critical Reviews in Food Science and Nutrition, 20(3), 173-248.

Fernández, P. P., Martino, M. N., Zaritzky, N. E., Guignon, B., \& Sanz, P. D. (2007). Effects of locust bean, xanthan and guar gums on the ice crystals of a sucrose solution frozen at high pressure. Food Hydrocolloids, 21(4), 507-515.

Fuchigami, M., Ogawa, N., \& Teramoto, A. (2002). Trehalose and hydrostatic pressure effects on the structure and sensory properties of frozen tofu (soybean curd). Innovative Food Science \& Emerging Technologies, 3(2), 139-147.

Guignon, B., Torrecilla, J. S., Otero, L., Ramos, A. M., Molina-García, A. D., \& Sanz, P. D. (2008). The initial freezing temperature of foods at high pressure. CRC Critical Reviews in Food Science and Nutrition, 48(4), 328-340.

Habib, B., \& Farid, M. (2006). Heat transfer and operating conditions for freeze concentration in a liquid-solid fluidized bed heat exchanger. Chemical Engineering and Processing, 45(8), 698-710.

Heneghan, A. F., Wilson, P. W., \& Haymet, A. D. J. (2002). Heterogeneous nucleation of supercooled water, and the effect of an added catalyst. Proceedings of the National Academy of Sciences of the United States of America, 99(15), 96319634.

Hernández, E., Raventós, M., Auleda, J. M., \& Ibarz, A. (2010). Freeze concentration of must in a pilot plant falling film cryoconcentrator. Innovative Food Science \& Emerging Technologies, 11(1), 130-136. 
Kanda, Y., Aoki, M., \& Kosugi, T. (1992). Freezing of tofu (soybean curd) by pressureshift freezing and its structure. Nippon Shokuhin Kogyo Gakkaishi, 39(7), 608614.

Kiani, H., \& Sun, D. W. (2011). Water crystallization and its importance to freezing of foods: A review. Trends in Food Science and Technology, 22(8), 407-426.

Kiparissides, C., Baltsas, A., Papadopoulos, S., Congalidis, J. P., Richards, J. R., Kelly, M. B., \& Ye, Y. (2005). Mathematical modeling of free-radical ethylene copolymerization in high-pressure tubular reactors. Industrial \& Engineering Chemistry Research, 44(8), 2592-2605.

Knorr, D., Schlüter, O., \& Heinz, V. (1998). Impact of high hydrostatic pressure on phase transition of foods. Food Technology, 52(9), 42-45.

Kulisiewicz, L., Kowalczyk, W., Baars, A., \& Delgado, A. (2007). Visualization of pressure-shift freezing and thawing of concentrated aqueous sucrose solutions. High Pressure Research, 27(2), 291-297.

LeBail, A., Chevalier, D., Mussa, D. M., \& Ghoul, M. (2002). High pressure freezing and thawing of foods: a review. International Journal of Refrigeration, 25(5), 504-513.

Lévy, J., Dumay, E., Kolodziejczyk, E., \& Cheftel, J. C. (1999). Freezing kinetics of a model oil-in-water emulsion under high pressure or by pressure release. Impact on ice crystals and oil droplets. Lebensmittel-Wissenschaft und-Technologie, 32(7), 396-405.

Martino, M. N., Otero, L., Sanz, P. D., \& Zaritzky, N. E. (1998). Size and location of ice crystals in pork frozen by high-pressure-assisted freezing as compared to classical methods. Meat Science, 50(3), 303-313.

Otero, L., Molina-García, A. D., \& Sanz, P. D. (2002). Some interrelated thermophysical properties of liquid water and ice. I. A user-friendly modeling review for food high-pressure processing. CRC Critical Reviews in Food Science and Nutrition, 42(4), 339-352.

Otero, L., Ousegui, A., Benet, G. U., de Elvira, C., Havet, M., Le Bail, A., \& Sanz, P. D. (2007). Modelling industrial scale high-pressure-low-temperature processes. Journal of Food Engineering, 83(2), 136-141.

Otero, L., \& Sanz, P. D. (2000). High-pressure shift freezing. Part 1. Amount of ice instantaneously formed in the process. Biotechnology Progress, 16(6), 10301036.

Otero, L., \& Sanz, P. D. (2006). High-pressure-shift freezing: Main factors implied in the phase transition time. Journal of Food Engineering, 72(4), 354-363.

Otero, L., Sanz, P. D., \& Guignon, B. (2009a). Método de crioconcentración de líquidos. Patent ES 2350428 A1. Spain.

Otero, L., Sanz, P. D., Guignon, B., \& Aparicio, C. (2009b). Experimental determination of the amount of ice instantaneously formed in high-pressure shift freezing. Journal of Food Engineering, 95(4), 670-676.

Petzold, G., \& Aguilera, J. M. (2009). Ice morphology: Fundamentals and technological applications in foods. Food Biophysics, 4(4), 378-396.

PT, G. M. (2010). Freeze concentration. Break barriers in liquid processing. http://www.gea-messo-pt.com/geacrystal/cmsdoc.nsf/WebDoc/webb7s6gze. Accessed 02/09/2011.

Rahman, M. S., Ahmed, M., \& Chen, X. D. (2006). Freezing-melting process and desalination: I. Review of the state-of-the-art. Separation \& Purification Reviews, 35(2), 59-96. 
Ramaswamy, R., Balasubramaniam, V. M., \& Sastry, S. K. (2007). Thermal conductivity of selected liquid foods at elevated pressures up to $700 \mathrm{MPa}$. Journal of Food Engineering, 83(3), 444-451.

Ramteke, R. S., Singh, N. I., Rekha, M. N., \& Eipeson, W. E. (1993). Methods for concentration of fruit juices: A critical evaluation. Journal of Food Science and Technology, 30(6), 391-402.

Reid, D. S. (1983). Fundamental physicochemical aspects of freezing. Food Technology, 37(4), 110-115.

Sanz, P. D., \& Otero, L. (2005). High-pressure freezing. In S. Da-Wen (Ed.), Emerging Technologies for Food Processing (pp. 627-652). London: Academic Press.

Saxholt, E., Christensen, A. T., Moller, A., Hartkopp, H. B., Hess Ygil, K., \& Hels, O. H. (2008). Danish Food Composition DataBank, revision 7. Department of Nutrition, National Food Institute, Technical University of Denmark. http://www.foodcomp.dk/. Accessed 02/09/2011.

Smith, C. E., \& Schwartzberg, H. G. (1985). Ice crystal size changes during ripening in freeze concentration. Biotechnology Progress, 1(2), 111-120.

Thiebaud, M., Dumay, E. M., \& Cheftel, J. C. (2002). Pressure-shift freezing of o/w emulsions: Influence of fructose and sodium alginate on undercooling, nucleation, freezing kinetics and ice crystal size distribution. Food Hydrocolloids, 16(6), 527-545.

Trieb, F., Karl, R., \& Moderer, R. (2007). Developments on flow rate and high pressure stability of peroxide dosing pumps for the chemical industry. AIDIC Conference Series, 8, 327-334.

Urrutia, G., Arabas, J., Autio, K., Brul, S., Hendrickx, M., Kakolewski, A., Knorr, D., Le Bail, A., Lille, M., Molina-García, A. D., Ousegui, A., Sanz, P. D., Shen, T., \& Van Buggenhout, S. (2007). SAFE ICE: Low-temperature pressure processing of foods: Safety and quality aspects, process parameters and consumer acceptance. Journal of Food Engineering, 83(2), 293-315.

Van Nistelrooij, M. (2005). Bridging the cost barrier to freeze concentration. Food \& Beverage Asia, April/May.

Verschuur, R. J., Scholz, R., Van Nistelrooij, M., \& Schreurs, B. (2002). Innovations in freeze concentration technology. In Proceedings of the 15th International Symposium on Industrial Crystallization (pp. 1035-1040). Sorrento, Italy.

Wagner, W., Saul, A., \& Pruss, A. (1994). International equations for the pressure along the melting and along the sublimation curve of ordinary water substance. Journal of Physical and Chemical Reference Data, 23(3), 515-525.

Werner, M., Baars, A., Werner, F., Eder, C., \& Delgado, A. (2007). Thermal conductivity of aqueous sugar solutions under high pressure. International Journal of Thermophysics, 28, 1161-1180.

Wilson, P. W., \& Haymet, A. D. J. (2009). Effect of solutes on the heterogeneous nucleation temperature of supercooled water: an experimental determination. Physical Chemistry Chemical Physics, 11(15), 2679-2682.

Wolfe, J., \& Bryant, G. (1999). Freezing, drying, and/or vitrification of membranesolute-water systems. Cryobiology, 39(2), 103-129.

Zhu, S., Le Bail, A., \& Ramaswamy, H. S. (2003). Ice crystal formation in pressure shift freezing of Atlantic salmon (Salmo salar) as compared to classical freezing methods. Journal of food processing and preservation., 27(6), 427-444.

Zhu, S., Le Bail, A., Ramaswamy, H. S., \& Chapleau, N. (2004). Characterization of ice crystals in pork muscle formed by pressure-shift freezing as compared with classical freezing methods. Journal of Food Science, 69(4), FEP190-FEP197. 
Zhu, S., Ramaswamy, H. S., \& Le Bail, A. (2005). Ice-crystal formation in gelatin gel during pressure shift versus conventional freezing. Journal of Food Engineering, 66(1), 69-76. 
TABLE 1 Rises in temperature (mean \pm standard deviation, ${ }^{\circ} \mathrm{C}$ ) recorded in orange juice samples, after compression, in PSN experiments at different pressure/temperature conditions.

\begin{tabular}{|c|c|c|c|c|c|}
\hline P (MPa) & $\mathrm{T}\left({ }^{\circ} \mathrm{C}\right)$ & 11.8 oBrix & 20 Brix & 30 Brix & 40 Brix \\
\hline 200 & -5 & $2.17 \pm 0.33$ & $3.53 \pm 0.06$ & $4.21 \pm 0.07$ & $4.54 \pm 0.13$ \\
\hline 350 & -5 & $5.41 \pm 0.33$ & $7.31 \pm 0.17$ & $7.94 \pm 0.13$ & $8.40 \pm 0.19$ \\
\hline 500 & -5 & $8.72 \pm 0.29$ & $10.54 \pm 0.38$ & $11.60 \pm 0.00$ & $12.72 \pm 0.95$ \\
\hline 700 & -5 & $9.96 \pm 0.24$ & $10.51 \pm 0.54$ & $13.58 \pm 0.50$ & $13.71 \pm 0.04$ \\
\hline 200 & -20 & $-0.46 \pm 0.25$ & $2.53 \pm 0.12$ & $3.16 \pm 0.32$ & $3.86 \pm 0.64$ \\
\hline 350 & -20 & $3.43 \pm 0.71$ & $5.17 \pm 0.66$ & $6.27 \pm 0.17$ & $6.87 \pm 0.21$ \\
\hline
\end{tabular}


TABLE 2 Extent of supercooling (mean \pm standard deviation, ${ }^{\circ} \mathrm{C}$ ) reached in orange juice samples, after expansion, in PSN experiments at different pressure/temperature conditions. *: Determination of the extent of supercooling was not feasible because nucleation took place very quickly, in $1 \mathrm{~s}$ or less after expansion.

\begin{tabular}{|c|c|c|c|c|c|}
\hline $\mathbf{P}(\mathrm{MPa})$ & $T\left({ }^{\circ} \mathrm{C}\right)$ & 11.8 oBrix & 20 Brix & 30 Brix & 40 Brix \\
\hline 200 & -5 & $6.31 \pm 0.01$ & $5.94 \pm 0.04$ & $4.88 \pm 0.25$ & $3.46 \pm 0.17$ \\
\hline 350 & -5 & $9.70 \pm 0.18$ & $9.23 \pm 0.26$ & $8.32 \pm 0.06$ & $6.89 \pm 0.00$ \\
\hline 500 & -5 & * & * & $10.34 \pm 0.09$ & $10.16 \pm 0.15$ \\
\hline 700 & -5 & * & * & * & $13.70 \pm 0.05$ \\
\hline 200 & -20 & * & * & * & * \\
\hline 350 & -20 & * & * & * & * \\
\hline
\end{tabular}


TABLE $3 \quad \mathrm{~T}_{\max }$ values (mean \pm standard deviation, ${ }^{\circ} \mathrm{C}$ ) reached in orange juice samples after expansion from different pressure/temperature conditions.

\begin{tabular}{|c|c|c|c|c|c|}
\hline$P(\mathrm{MPa})$ & $\mathbf{T}\left({ }^{\circ} \mathbf{C}\right)$ & $11.8^{\circ}$ Brix & 20 oBrix & $30 \stackrel{\circ}{ }$ Brix & 40 oBrix \\
\hline 200 & -5 & $-1.25 \pm 0.00$ & $-2.38 \pm 0.04$ & $-4.27 \pm 0.05$ & --- \\
\hline 350 & -5 & $-1.35 \pm 0.05$ & $-2.59 \pm 0.07$ & $-4.30 \pm 0.12$ & $-7.01 \pm 0.04$ \\
\hline 500 & -5 & $-1.52 \pm 0.03$ & $-2.67 \pm 0.03$ & $-4.53 \pm 0.03$ & $-7.51 \pm 0.10$ \\
\hline 700 & -5 & $-1.61 \pm 0.06$ & $-2.79 \pm 0.05$ & $-4.95 \pm 0.05$ & $-7.84 \pm 0.06$ \\
\hline 200 & -20 & $-1.75 \pm 0.08$ & $-3.02 \pm 0.09$ & $-5.17 \pm 0.03$ & $-8.25 \pm 0.00$ \\
\hline 350 & -20 & $-1.91 \pm 0.12$ & $-3.16 \pm 0.08$ & $-5.53 \pm 0.03$ & $-8.93 \pm 0.08$ \\
\hline
\end{tabular}




\section{FIGURE CAPTIONS}

Figure 1 Pressure and temperature coordinates from which expansions were made in the pressure-shift nucleation experiments realized. Solid lines represent the phase diagram of pure water (Wagner et al., 1994). Dotted lines represent the phase diagrams estimated in this paper for the different orange juices employed.

Figure 2 Pressure and temperature evolution in a $11.8{ }^{\circ}$ Brix orange juice sample during a representative pressure-shift nucleation experiment at $200 \mathrm{MPa} /-5 \stackrel{\circ}{\circ} \mathrm{C} . \mathrm{T}_{\min }$ and $T_{\mathrm{fp}}\left([\mathrm{OJ}]_{\mathrm{F}}\right)$ represent the minimum and the maximum temperatures, respectively, reached in the sample after expansion.

Figure 3 Pressure-temperature evolution in orange juice samples of different concentration during PSN experiments at $350 \mathrm{MPa}$ and different temperatures. (a) $350 \mathrm{MPa} /-5 \stackrel{\circ}{\circ} \mathrm{C}$ in a 11.8 Brix orange juice sample: Step 1-2: Compression, step 2-3: Pressure holding step, step 3-4: Expansion, step: 4-5: Nucleation; (b) $350 \mathrm{MPa} /-5{ }^{\circ} \mathrm{C}$ in a $40{ }^{\circ}$ Brix orange juice sample; (c) $350 \mathrm{MPa} /-20{ }^{\circ} \mathrm{C}$ in a 11.8 Brix orange juice sample; and (d) $350 \mathrm{MPa} /-20 \stackrel{\circ}{\circ}$ in a $40{ }^{\circ}$ Brix orange juice sample. Solid bold lines represent the phase diagram of the orange juices employed. $\Delta T$ represents the extent of supercooling reached after expansion.

Figure 4 Temperature evolution in orange juice samples after expansions from different pressure/temperature conditions. (a) $200 \mathrm{MPa} /-5{ }^{\circ} \mathrm{C}$; (b) $350 \mathrm{MPa} /-5 \stackrel{\circ}{\circ} \mathrm{C}$; (c) 200 $\mathrm{MPa} /-20 \stackrel{\circ}{\circ}$; and (d) $350 \mathrm{MPa} /-20 \stackrel{\circ}{\circ}$. Black points in the graphs represent the instant immediately previous to expansion. $T_{\min }$ and $T_{\max }$ represent the minimum and the maximum temperatures, respectively, reached in the sample after expansion.

Figure 5 Final concentration (mean values \pm standard deviation, ${ }^{\circ} B r i x$ ) reached in different orange juice samples after pressure-shift nucleation from different pressure and temperature conditions ( $\left.\square:-5{ }^{\circ} \mathrm{C} ; \square:-20 \stackrel{\circ}{\circ} \mathrm{C}\right)$.

Figure 6 Final concentration (Bix) reached in orange juice samples of different concentration after pressure-shift nucleation from different pressure and temperature conditions. (I): Values (mean \pm standard deviation values) calculated from equation (1) using the experimental $\mathrm{T}_{\max }$ data; (-): Values predicted by the model developed in this paper for experiments performed at -5 ${ }^{\circ} \mathrm{C}$; and $(\cdots)$ : Values predicted by the model developed in this paper for experiments at $-20 \stackrel{\circ}{ } \mathrm{C}$. 
Figure 7 Microphotographs taken in $11.8{ }^{\circ}$ Brix orange juice samples after pressure-shift nucleation from different pressure and temperature conditions.

Figure $8 \quad$ Ice crystal size distributions in $11.8^{\circ}$ Brix orange juice samples after pressureshift nucleation from different pressure and temperature conditions.

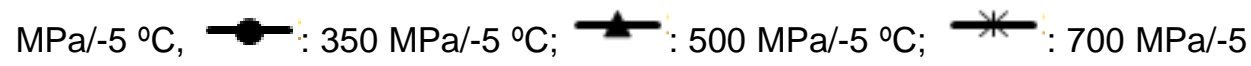
$\stackrel{\circ}{\circ} ;-\square-200 \mathrm{MPa} /-20 \stackrel{\circ}{\circ}$ and $\frown-$ - $350 \mathrm{MPa} /-20 \stackrel{\circ}{\circ}$.

Figure 9 Implementation of pressure-shift nucleation in a freeze concentration process 
FIGURE 1

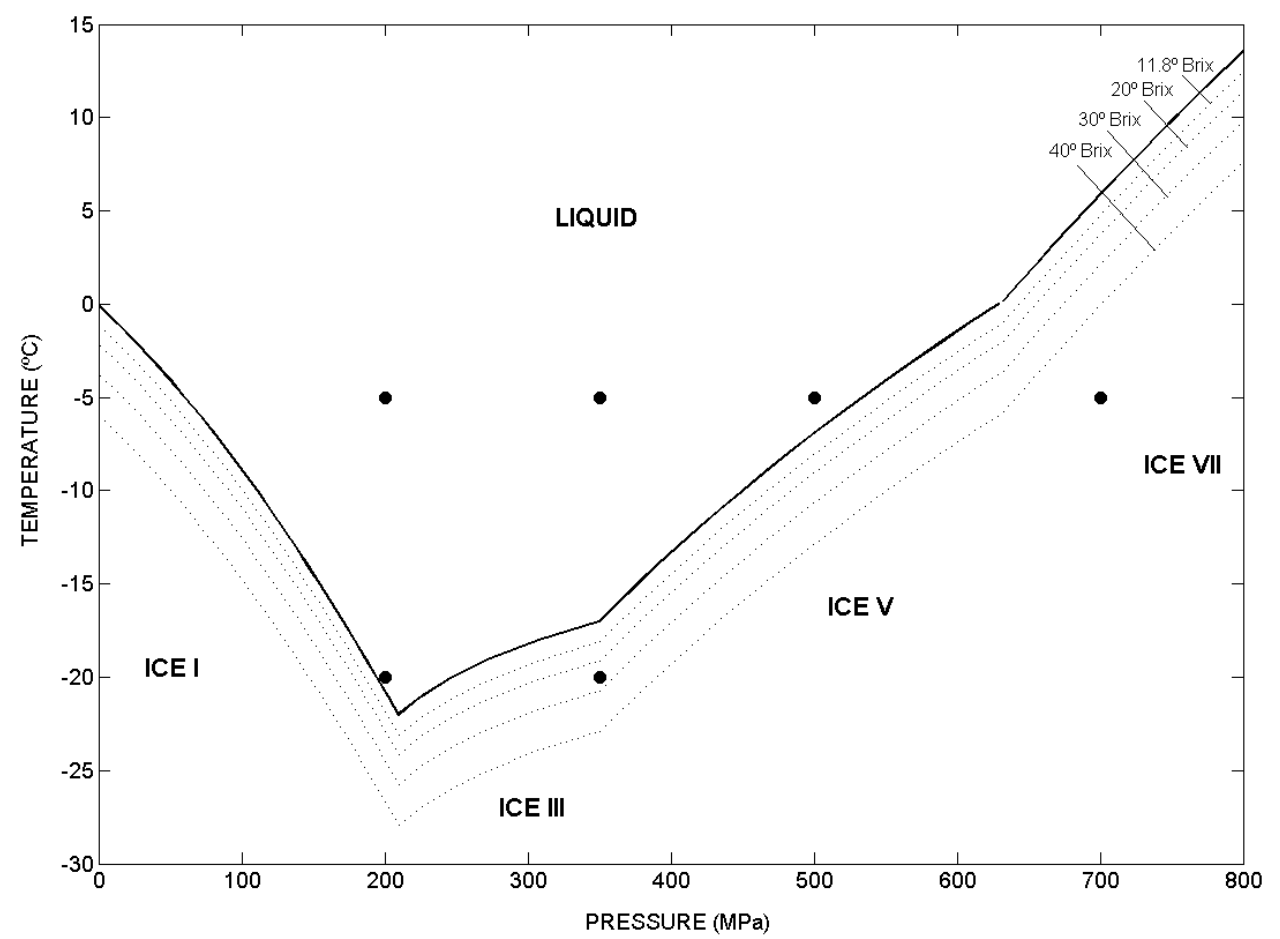


FIGURE 2
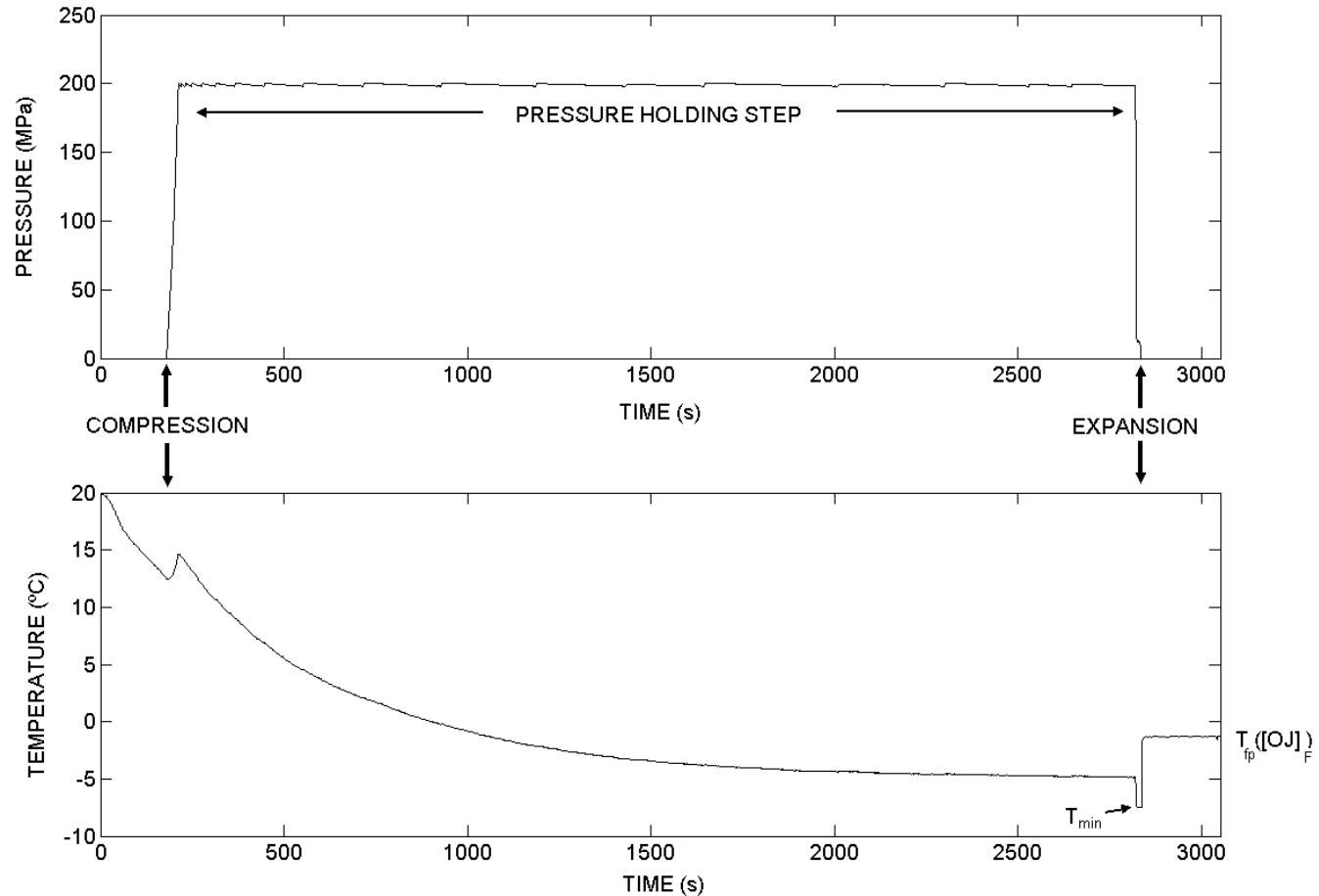


\section{FIGURE 3}

a)

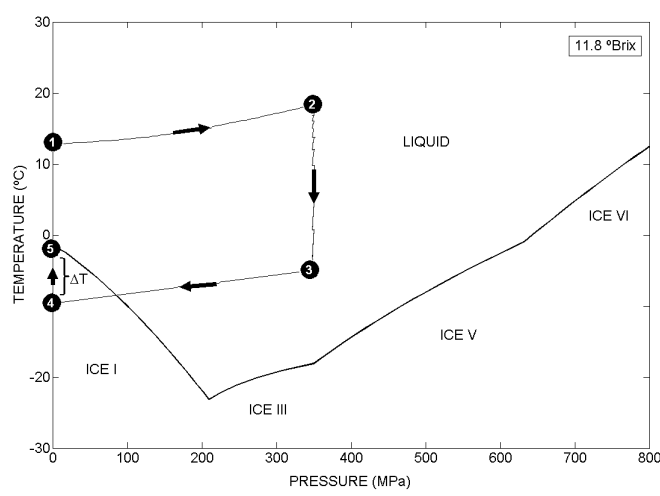

c)

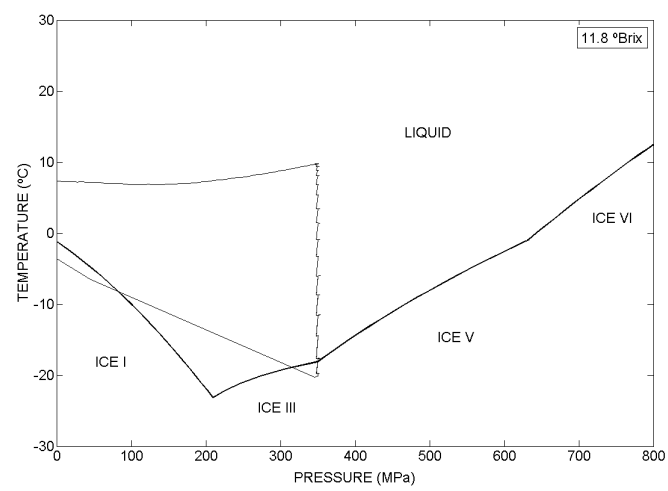

b)

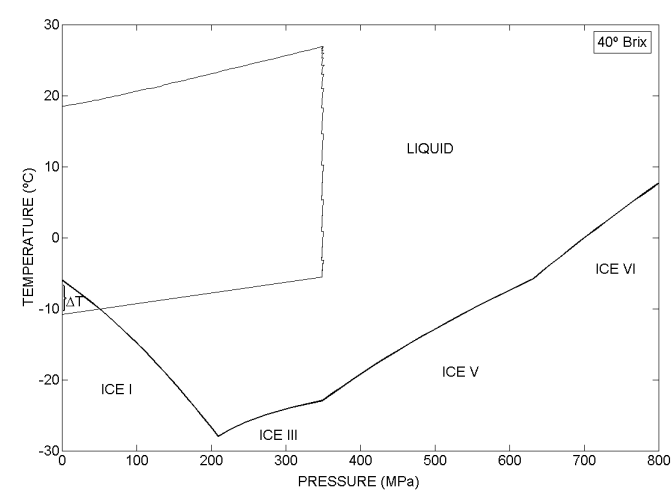

d)

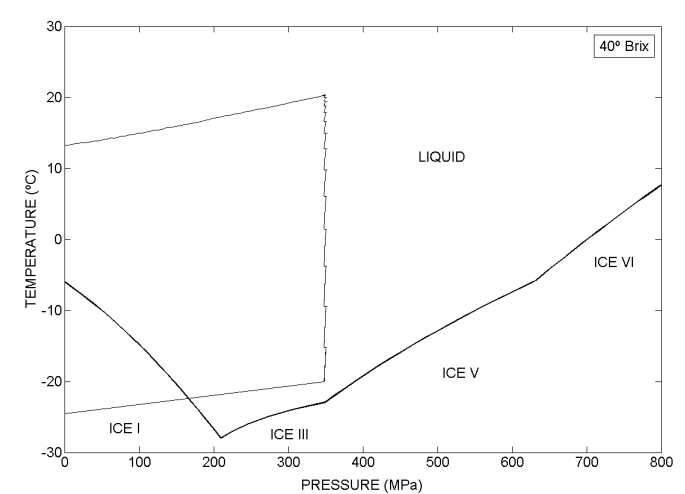




\section{FIGURE 4}

a)

c)
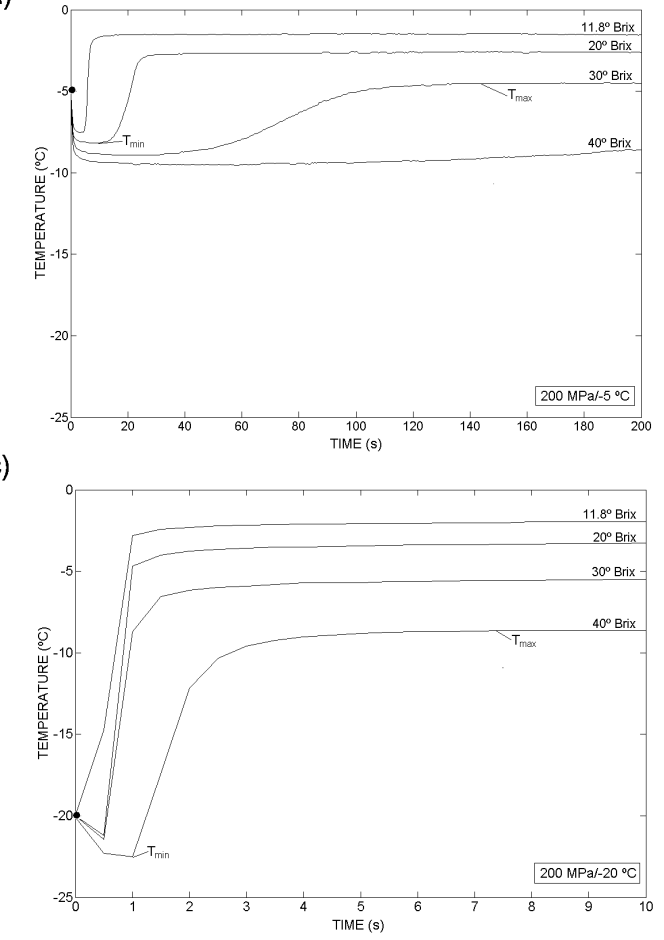

b)

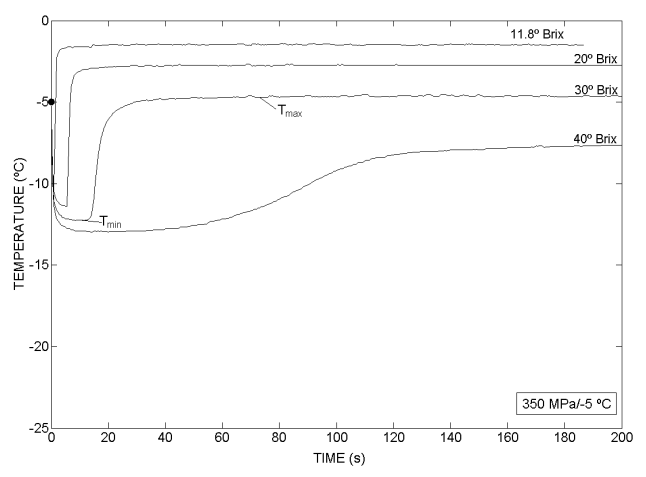

d)

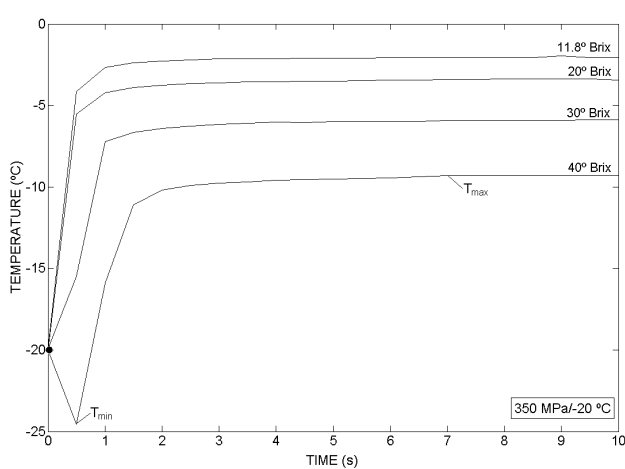




\section{FIGURE 5}

a)

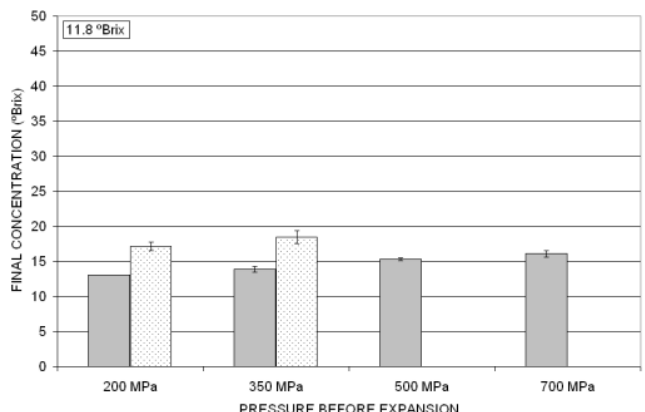

c)

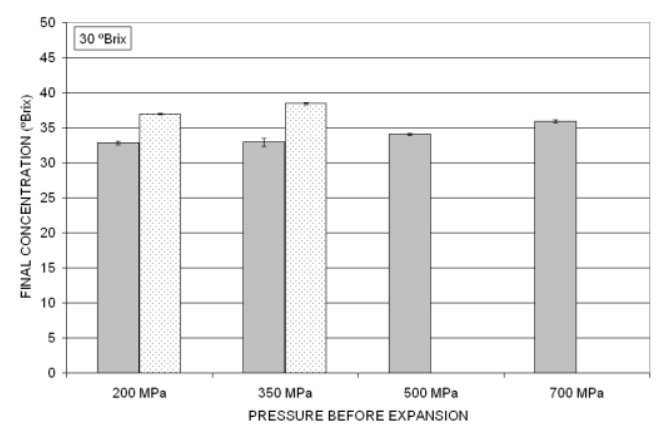

b)

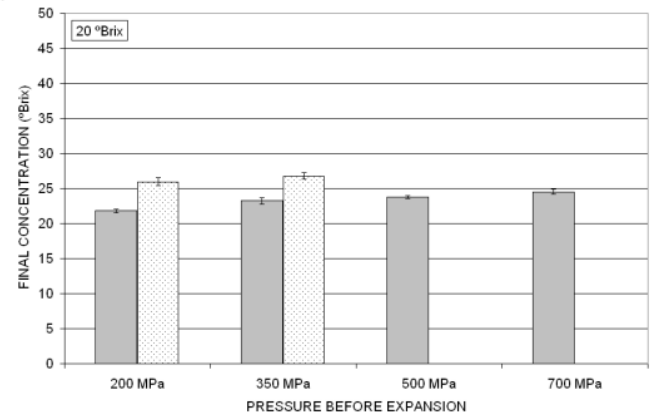

d)

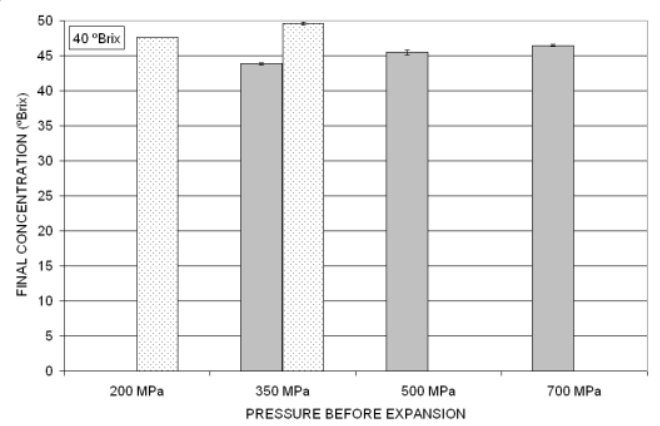


FIGURE 6

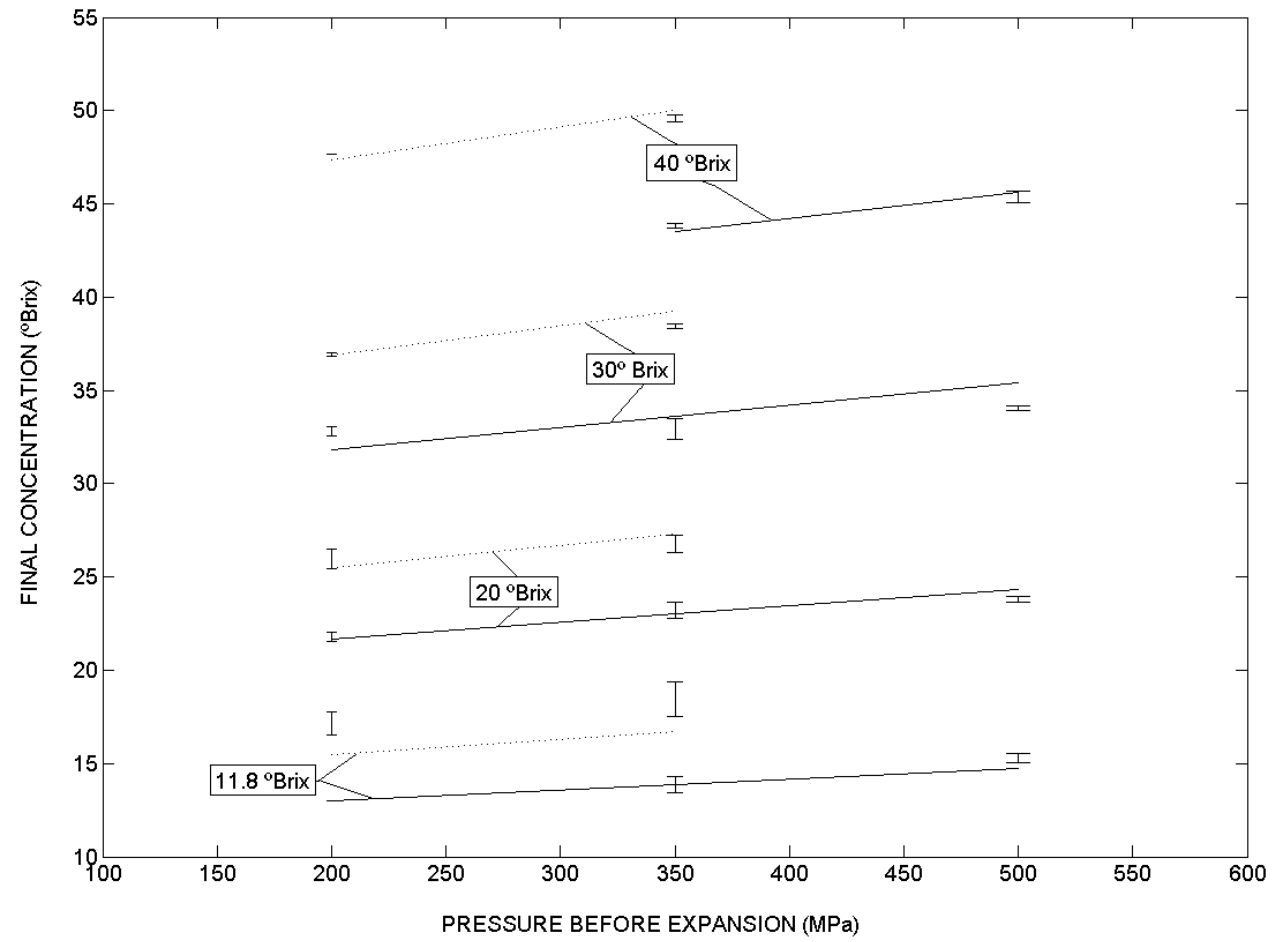


FIGURE 7
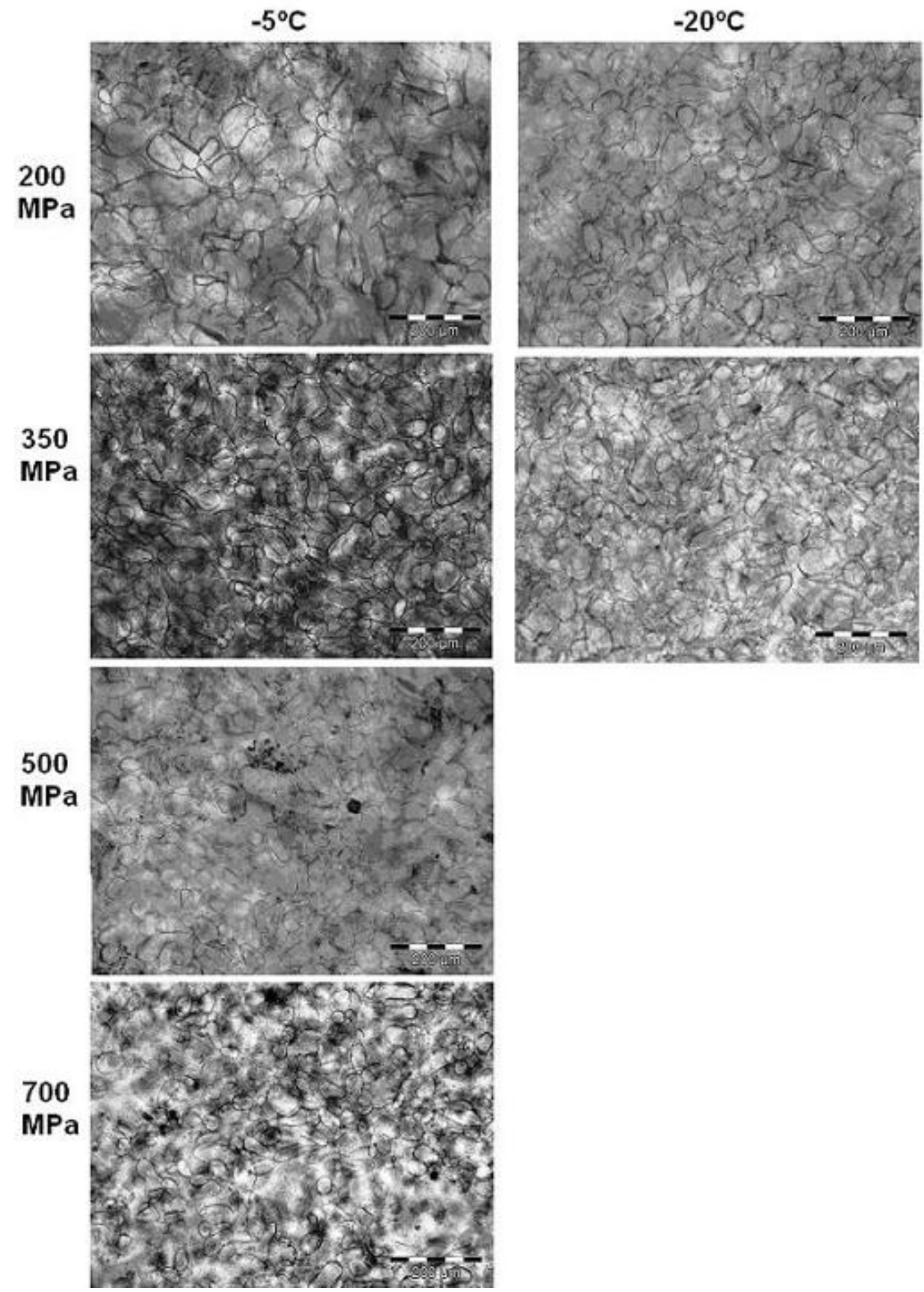
FIGURE 8

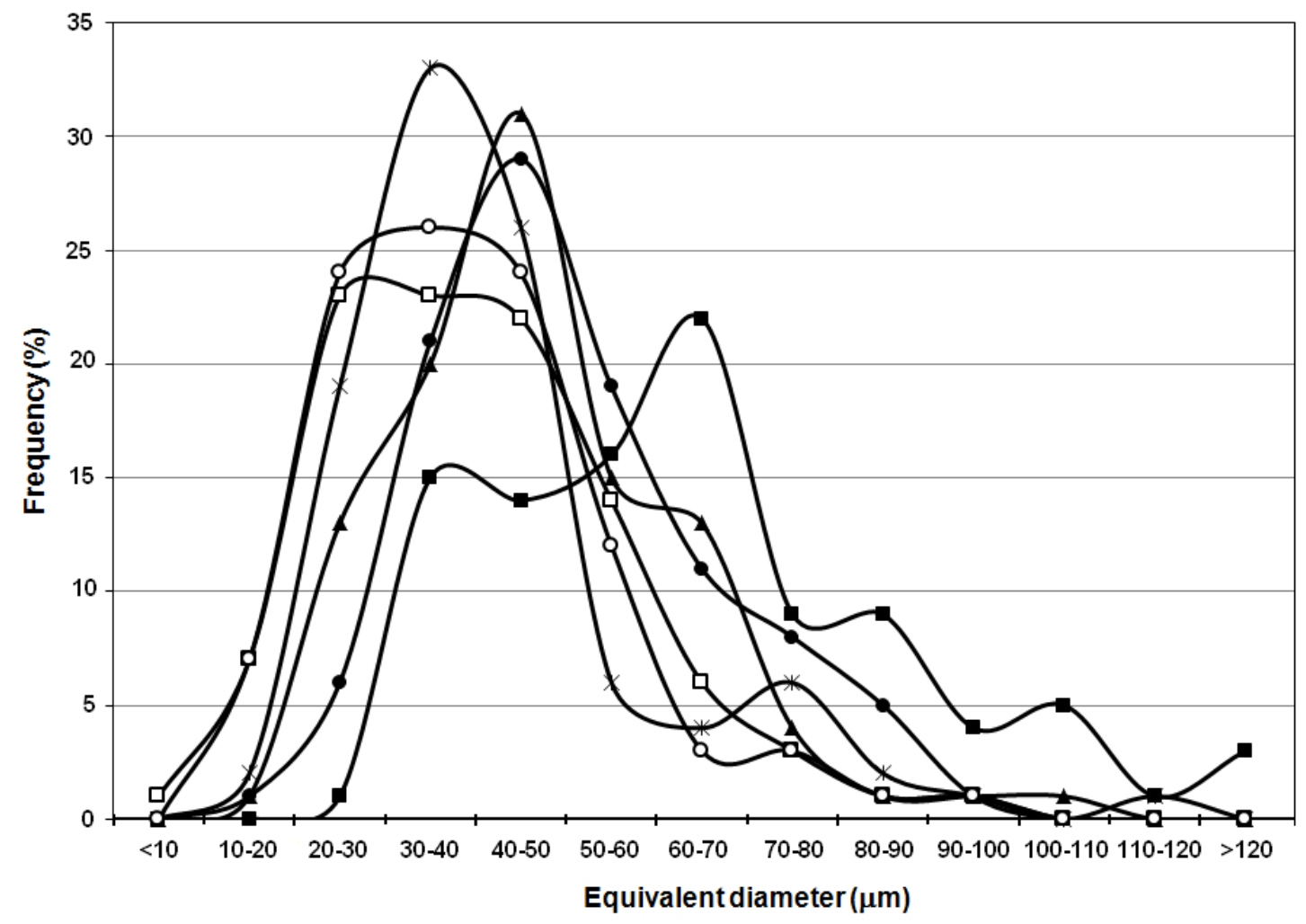




\section{FIGURE 9}

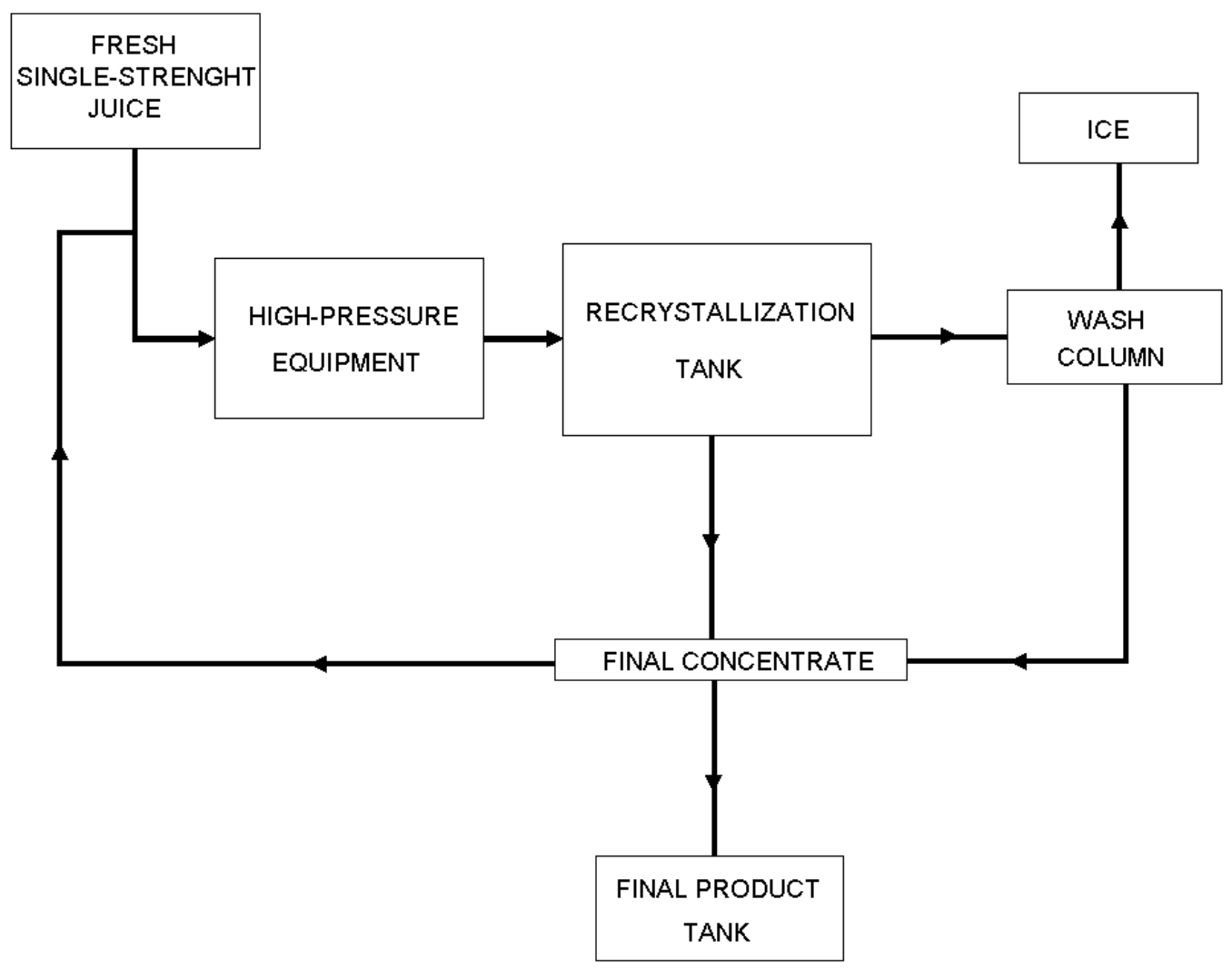

